\title{
Classes and equivalence of linear sets in $\mathrm{PG}\left(1, q^{n}\right)$
}

\author{
Bence Csajbók, Giuseppe Marino and Olga Polverino*
}

\begin{abstract}
The equivalence problem of $\mathbb{F}_{q}$-linear sets of rank $n$ of $\mathrm{PG}\left(1, q^{n}\right)$ is investigated, also in terms of the associated variety, projecting configurations, $\mathbb{F}_{q}$-linear blocking sets of Rédei type and MRD-codes. We call an $\mathbb{F}_{q}$-linear set $L_{U}$ of rank $n$ in $\mathrm{PG}\left(W, \mathbb{F}_{q^{n}}\right)=\mathrm{PG}\left(1, q^{n}\right)$ simple if for any $n$-dimensional $\mathbb{F}_{q}$-subspace $V$ of $W, L_{V}$ is $\operatorname{P\Gamma L}\left(2, q^{n}\right)$-equivalent to $L_{U}$ only when $U$ and $V$ lie on the same orbit of $\Gamma \mathrm{L}\left(2, q^{n}\right)$. We prove that $U=\left\{\left(x, \operatorname{Tr}_{q^{n} / q}(x)\right): x \in \mathbb{F}_{q^{n}}\right\}$ defines a simple $\mathbb{F}_{q^{-}}$-linear set for each $n$. We provide examples of non-simple linear sets not of pseudoregulus type for $n>4$ and we prove that all $\mathbb{F}_{q}$-linear sets of rank 4 are simple in $\mathrm{PG}\left(1, q^{4}\right)$.
\end{abstract}

\section{Introduction}

Linear sets are natural generalizations of subgeometries. Let $\Lambda=\operatorname{PG}\left(W, \mathbb{F}_{q^{n}}\right)$ $=P G\left(r-1, q^{n}\right)$, where $W$ is a vector space of dimension $r$ over $\mathbb{F}_{q^{n}}$. A point set $L$ of $\Lambda$ is said to be an $\mathbb{F}_{q}$-linear set of $\Lambda$ of rank $k$ if it is defined by the non-zero vectors of a $k$-dimensional $\mathbb{F}_{q}$-vector subspace $U$ of $W$, i.e.

$$
L=L_{U}=\left\{\langle\mathbf{u}\rangle_{\mathbb{F}_{q^{n}}}: \mathbf{u} \in U \backslash\{\mathbf{0}\}\right\} .
$$

The maximum field of linearity of an $\mathbb{F}_{q^{-}}$linear set $L_{U}$ is $\mathbb{F}_{q^{t}}$ if $t \mid n$ is the largest integer such that $L_{U}$ is an $\mathbb{F}_{q^{t}}$-linear set. In the recent years, starting from the paper [20] by Lunardon, linear sets have been used to construct or characterize various objects in finite geometry, such as blocking sets and multiple blocking sets in finite projective spaces, two-intersection sets in finite

${ }^{*}$ The research was supported by Ministry for Education, University and Research of Italy MIUR (Project PRIN 2012 "Geometrie di Galois e strutture di incidenza") and by the Italian National Group for Algebraic and Geometric Structures and their Applications (GNSAGA - INdAM). The first author was partially supported by the János Bolyai Research Scholarship of the Hungarian Academy of Sciences and by OTKA Grant No. K 124950 . 
projective spaces, translation spreads of the Cayley Generalized Hexagon, translation ovoids of polar spaces, semifield flocks and finite semifields. For a survey on linear sets we refer the reader to [27], see also [16].

One of the most natural questions about linear sets is their equivalence. Two linear sets $L_{U}$ and $L_{V}$ of PG $\left(r-1, q^{n}\right)$ are said to be PГL-equivalent (or simply equivalent) if there is an element $\varphi$ in $\operatorname{P\Gamma L}\left(r, q^{n}\right)$ such that $L_{U}^{\varphi}=L_{V}$. In the applications it is crucial to have methods to decide whether two linear sets are equivalent or not. For $f \in \Gamma \mathrm{L}\left(r, q^{n}\right)$ we have $L_{U^{f}}=L_{U}^{\varphi_{f}}$, where $\varphi_{f}$ denotes the collineation of $\operatorname{PG}\left(W, \mathbb{F}_{q^{n}}\right)$ induced by $f$. It follows that if $U$ and $V$ are $\mathbb{F}_{q^{-}}$-subspaces of $W$ belonging to the same orbit of $\Gamma \mathrm{L}\left(r, q^{n}\right)$, then $L_{U}$ and $L_{V}$ are equivalent. The above condition is only sufficient but not necessary to obtain equivalent linear sets. This follows also from the fact that $\mathbb{F}_{q}$-subspaces of $W$ with different ranks can define the same linear set, for example $\mathbb{F}_{q^{-}}$-linear sets of $\mathrm{PG}\left(r-1, q^{n}\right)$ of rank $k \geq r n-n+1$ are all the same: they coincide with $\mathrm{PG}\left(r-1, q^{n}\right)$. As it was showed recently in [7], if $r=2$, then there exist $\mathbb{F}_{q^{-}}$-subspaces of $W$ of the same rank $n$ but on different orbits of $\Gamma \mathrm{L}\left(2, q^{n}\right)$ defining the same linear set of $\mathrm{PG}\left(1, q^{n}\right)$.

This observation motivates the following definition. An $\mathbb{F}_{q}$-linear set $L_{U}$ of $\mathrm{PG}\left(W, \mathbb{F}_{q^{n}}\right)=\mathrm{PG}\left(r-1, q^{n}\right)$ with maximum field of linearity $\mathbb{F}_{q}$ is called simple if for each $\mathbb{F}_{q}$-subspace $V$ of $W, L_{U}=L_{V}$ only if $U$ and $V$ are in the same orbit of $\Gamma \mathrm{L}\left(r, q^{n}\right)$ or, equivalently, if for each $\mathbb{F}_{q}$-subspace $V$ of $W$, $L_{V}$ is $\operatorname{P\Gamma L}\left(r, q^{n}\right)$-equivalent to $L_{U}$ only if $U$ and $V$ are in the same orbit of $\Gamma \mathrm{L}\left(r, q^{n}\right)$.

Natural examples of simple linear sets are the subgeometries (cf. 19, Theorem 2.6] and [15, Section 25.5]). In [6] it was proved that $\mathbb{F}_{q}$-linear sets of rank $n+1$ of $\operatorname{PG}\left(2, q^{n}\right)$ admitting $(q+1)$-secants are simple. This allowed the authors to translate the question of equivalence to the study of the orbits of the stabilizer of a subgeometry on subspaces and hence to obtain the complete classification of $\mathbb{F}_{q}$-linear blocking sets in $\operatorname{PG}\left(2, q^{4}\right)$. Until now, the only known examples of non-simple linear sets are those of pseudoregulus type of $\mathrm{PG}\left(1, q^{n}\right)$ for $n \geq 5$ and $n \neq 6$, see [7.

In this paper we focus on linear sets of rank $n$ of $\operatorname{PG}\left(1, q^{n}\right)$. We first introduce a method which can be used to find non-simple linear sets of rank $n$ of $\mathrm{PG}\left(1, q^{n}\right)$. Let $L_{U}$ be a linear set of rank $n$ of $\operatorname{PG}\left(W, \mathbb{F}_{q^{n}}\right)=\operatorname{PG}\left(1, q^{n}\right)$ and let $\beta$ be a non-degenerate alternating form of $W$. Denote by $\perp$ the orthogonal complement map induced by $\operatorname{Tr}_{q^{n} / q} \circ \beta$ on $W$ (considered as an

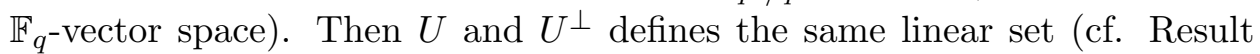
2.1 and if $U$ and $U^{\perp}$ lie on different orbits of $\Gamma L\left(W, \mathbb{F}_{q^{n}}\right)$, then $L_{U}$ is nonsimple. Using this approach we show that there are non-simple linear sets 
of rank $n$ of $\mathrm{PG}\left(1, q^{n}\right)$ for $n \geq 5$, not of pseudoregulus type (cf. Proposition 3.10). Contrary to what we expected initially, simple linear sets are harder to find than non-simple linear sets. We prove that the linear set of $\operatorname{PG}\left(1, q^{n}\right)$ defined by the trace function is simple (cf. Theorem 3.7). We also show that linear sets of rank $n$ of $\mathrm{PG}\left(1, q^{n}\right)$ are simple for $n \leq 4$ (cf. Theorem 4.5).

Moreover, in $\mathrm{PG}\left(1, q^{n}\right)$ we extend the definition of simple linear sets and introduce the $\mathcal{Z}(\Gamma \mathrm{L})$-class and the $\Gamma$-class for linear sets of rank $n$. In Section 5 we point out the meaning of these classes in terms of equivalence of the associated blocking sets, MRD-codes and projecting configurations.

\section{Definitions and preliminary results}

\subsection{Dual linear sets with respect to a symplectic polarity of a line}

For $\alpha \in \mathbb{F}_{q^{n}}$ and a divisor $h$ of $n$ we will denote by $\operatorname{Tr}_{q^{n} / q^{h}}(\alpha)$ the trace of $\alpha$ over the subfield $\mathbb{F}_{q^{h}}$, that is, $\operatorname{Tr}_{q^{n} / q^{h}}(\alpha)=\alpha+\alpha^{q^{h}}+\ldots+\alpha^{q^{n-h}}$. By $\mathrm{N}_{q^{n} / q^{h}}(\alpha)$ we will denote the norm of $\alpha$ over the subfield $\mathbb{F}_{q^{h}}$, that is, $\mathrm{N}_{q^{n} / q^{h}}(\alpha)=\alpha^{1+q^{h}+\ldots+q^{n-h}}$. Since in the paper we will use only norms over $\mathbb{F}_{q}$, the function $\mathrm{N}_{q^{n} / q}$ will be denoted simply by $\mathrm{N}$.

Starting from a linear set $L_{U}$ of $\operatorname{PG}\left(r, q^{n}\right)$ and using a polarity $\tau$ of the space it is always possible to construct another linear set, which is called dual linear set of $L_{U}$ with respect to the polarity $\tau$ (see [27]). In particular, let $L_{U}$ be an $\mathbb{F}_{q}$-linear set of rank $n$ of a line $\mathrm{PG}\left(W, \mathbb{F}_{q^{n}}\right)$ and let $\beta: W \times W \longrightarrow \mathbb{F}_{q^{n}}$

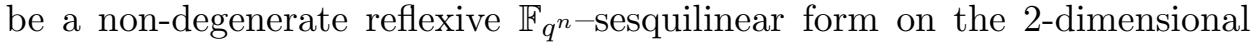
vector space $W$ over $\mathbb{F}_{q^{n}}$ determining a polarity $\tau$. The map $\operatorname{Tr}_{q^{n} / q} \circ \beta$ is a non-degenerate reflexive $\mathbb{F}_{q}$-sesquilinear form on $W$, when $W$ is regarded as a $2 n$-dimensional vector space over $\mathbb{F}_{q}$ (see [14]).

Let $\perp_{\beta}$ and $\perp_{\beta}^{\prime}$ be the orthogonal complement maps defined by $\beta$ and

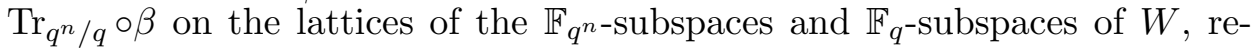
spectively. The dual linear set of $L_{U}$ with respect to the polarity $\tau$ is the $\mathbb{F}_{q}$-linear set of rank $n$ of $\mathrm{PG}\left(W, \mathbb{F}_{q^{n}}\right)$ defined by the orthogonal complement $U^{\perp^{\prime}}$ and it will be denoted by $L_{U}^{\tau}$. Also, up to projective equivalence, such a linear set does not depend on $\tau$ [27, Proposition 2.5].

For a point $P=\langle\mathbf{z}\rangle_{\mathbb{F}_{q^{n}}} \in \mathrm{PG}\left(W, \mathbb{F}_{q^{n}}\right)$ the weight of $P$ with respect to the linear set $L_{U}$ is $w_{L_{U}}(P):=\operatorname{dim}_{q}\left(\langle\mathbf{z}\rangle_{\mathbb{F}_{q^{n}}} \cap U\right)$.

Result 2.1. From [27, Property 2.6] (with $r=2, s=0$ and $t=n$ ) it can be easily seen that if $L_{U}$ is an $\mathbb{F}_{q}$-linear set of rank $n$ of a line $\mathrm{PG}\left(1, q^{n}\right)$ and $L_{U}^{\tau}$ 
is its dual linear set with respect to a polarity $\tau$, then $w_{L_{U}^{\tau}}\left(P^{\tau}\right)=w_{L_{U}}(P)$ for each point $P \in \mathrm{PG}\left(1, q^{n}\right)$. If $\tau$ is a symplectic polarity of a line $\mathrm{PG}\left(1, q^{n}\right)$, then $P^{\tau}=P$ and hence $L_{U}=L_{U}^{\tau}=L_{U^{\perp^{\prime}}}$.

\section{$2.2 \quad \mathbb{F}_{q}$-linear sets of $\mathrm{PG}\left(1, q^{n}\right)$ of class $r$}

In this paper we investigate the equivalence of $\mathbb{F}_{q}$-linear sets of rank $n$ of the projective line $\operatorname{PG}\left(W, \mathbb{F}_{q^{n}}\right)=\operatorname{PG}\left(1, q^{n}\right)$. The first step is to determine the $\mathbb{F}_{q}$-vector subspaces of $W$ defining the same linear set. This motivates the definition of the $\mathcal{Z}(\Gamma L)$-class and $\Gamma$ L-class of a linear set $L_{U}$ of $\operatorname{PG}\left(1, q^{n}\right)$ (cf. Definitions 2.4 and 2.5). The next proposition relies on the characterization of functions over $\mathbb{F}_{q}$ determining few directions. It states that the $\mathbb{F}_{q}$-rank of $L_{U}$ of $\mathrm{PG}\left(1, q^{n}\right)$ is uniquely defined when the maximum field of linearity of $L_{U}$ is $\mathbb{F}_{q}$. This will allow us to state our definitions and results without further conditions on the rank of the corresponding $\mathbb{F}_{q}$-subspaces.

For an $\mathbb{F}_{q}$ to $\mathbb{F}_{q}$ function $f$, the set of directions determined by $f$ is

$$
D_{f}:=\left\{\frac{f(x)-f(y)}{x-y}: x, y \in \mathbb{F}_{q^{n}}, x \neq y\right\} .
$$

Theorem 2.2 (Ball et al. [3] and Ball [1]). Let $f$ be a function from $\mathbb{F}_{q}$ to $\mathbb{F}_{q}, q=p^{h}$, and let $N$ be the number of directions determined by $f$. Let $s=p^{e}$ be maximal such that any line with a direction determined by $f$ that is incident with a point of the graph of $f$ is incident with a multiple of $s$ points of the graph of $f$. Then one of the following holds.

1. $s=1$ and $(q+3) / 2 \leq N \leq q+1$,

2. $e \mid h, q / s+1 \leq N \leq(q-1) /(s-1)$,

3. $s=q$ and $N=1$.

Moreover if $s>2$, then the graph of $f$ is $\mathbb{F}_{s}$-linear.

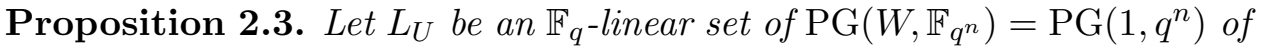
rank $n$. The maximum field of linearity of $L_{U}$ is $\mathbb{F}_{q^{d}}$, where

$$
d=\min \left\{w_{L_{U}}(P): P \in L_{U}\right\} .
$$

If the maximum field of linearity of $L_{U}$ is $\mathbb{F}_{q}$, then the rank of $L_{U}$ as an $\mathbb{F}_{q^{-}}$ linear set is uniquely defined, i.e. for each $\mathbb{F}_{q}$-subspace $V$ of $W$ if $L_{U}=L_{V}$, then $\operatorname{dim}_{q}(V)=n$. 
Proof. We first note that since $L_{U}$ is an $\mathbb{F}_{q^{-}}$linear set of $\mathrm{PG}\left(1, q^{n}\right)$ of rank $n$, then $\left|L_{U}\right| \leq\left(q^{n}-1\right) /(q-1)$ and hence $L_{U} \neq \operatorname{PG}\left(1, q^{n}\right)$.

Since the action of $\Gamma \mathrm{L}\left(2, q^{n}\right)$ preserves the maximum field of linearity and the weight of points, we can assume, up to the action of $\Gamma \mathrm{L}\left(2, q^{n}\right)$, that $U=\left\{(x, f(x)): x \in \mathbb{F}_{q^{n}}\right\}$ for some $q$-polynomial $f$ over $\mathbb{F}_{q^{n}}$. Since $f$ is linear, $\left|L_{U}\right|$ is the size of the set of directions determined by $f$. Also, a line $\ell$ with slope $m$ meets the graph of $f$ in $q^{t}$ points, where $t=w_{L_{U}}\left(\langle(1, m)\rangle_{\mathbb{F}_{q^{n}}}\right)$, i.e. $\left|\left\{z \in \mathbb{F}_{q^{n}}^{*}: f(z) / z=m\right\}\right|=q^{t}-1$.

Let $d=\min \left\{w_{L_{U}}(P): P \in L_{U}\right\}$. If $q=p^{e}, p$ prime, then $p^{d e}$ is the largest $p$-power such that every line with a determined direction that meets the graph of $f$ meets the graph of $f$ in a multiple of $s=p^{d e}$ points. Then Theorem 2.2 yields that either $s=q^{n}$ and $f(x)=\lambda x$ for some $\lambda \in \mathbb{F}_{q^{n}}$, or $\mathbb{F}_{q^{d}}$ is a proper subfield of $\mathbb{F}_{q^{n}}$ and

$$
q^{n-d}+1 \leq\left|L_{U}\right| \leq \frac{q^{n}-1}{q^{d}-1}
$$

Moreover, if $q^{d}>2$, then $f$ is $\mathbb{F}_{q^{d}}$-linear. In our case we already know that

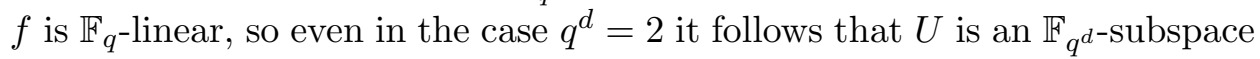
of $W$ and hence $L_{U}$ is an $\mathbb{F}_{q^{d}}$-linear set.

We show that $\mathbb{F}_{q^{d}}$ is the maximum field of linearity of $L_{U}$. Suppose, contrary to our claim, that $L_{U}$ is $\mathbb{F}_{q^{r}}$-linear of rank $z$ for some $r>d$. Then $L_{U}$ is also $\mathbb{F}_{q}$-linear of rank $r z$. It follows that $r z \leq n$ since otherwise $L_{U}=\operatorname{PG}\left(1, q^{n}\right)$. Then for the size of $L_{U}$ we get $\left|L_{U}\right| \leq\left(q^{r z}-1\right) /\left(q^{r}-1\right) \leq$ $\left(q^{n}-1\right) /\left(q^{r}-1\right)$, and this number is less than the lower bound in (1). This shows $r=d$.

Now suppose that $\mathbb{F}_{q}$ is the maximum field of linearity of $L_{U}$ and let $V$ be an $r$-dimensional $\mathbb{F}_{q}$-subspace of $W$ such that $L_{U}=L_{V}$. We cannot have $r>n$ since $L_{U} \neq \mathrm{PG}\left(1, q^{n}\right)$. Suppose, contrary to our claim, that $r \leq n-1$. Then $\left|L_{U}\right| \leq\left(q^{n-1}-1\right) /(q-1)$ contradicting (1) which gives $q^{n-1}+1 \leq\left|L_{U}\right|$. This concludes the proof.

Now we can give the following definitions of classes of an $\mathbb{F}_{q}$-linear set of a line.

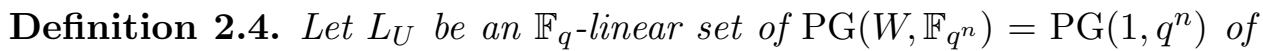
rank $n$ with maximum field of linearity $\mathbb{F}_{q}$. We say that $L_{U}$ is of $\mathcal{Z}(\Gamma \mathrm{L})$-class $r$ if $r$ is the largest integer such that there exist $\mathbb{F}_{q}$-subspaces $U_{1}, U_{2}, \ldots, U_{r}$ of $W$ with $L_{U_{i}}=L_{U}$ for $i \in\{1,2, \ldots, r\}$ and $U_{i} \neq \lambda U_{j}$ for each $\lambda \in \mathbb{F}_{q^{n}}^{*}$ and for each $i \neq j, i, j \in\{1,2, \ldots, r\}$. 


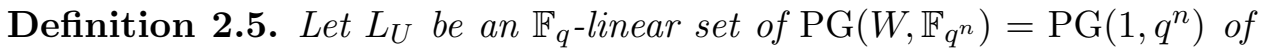
rank $n$ with maximum field of linearity $\mathbb{F}_{q}$. We say that $L_{U}$ is of $\Gamma \mathrm{L}$-class $s$ if $s$ is the largest integer such that there exist $\mathbb{F}_{q}$-subspaces $U_{1}, U_{2}, \ldots, U_{s}$ of $W$ with $L_{U_{i}}=L_{U}$ for $i \in\{1,2, \ldots, s\}$ and there is no $f \in \Gamma \mathrm{L}\left(2, q^{n}\right)$ such that $U_{i}=U_{j}^{f}$ for each $i \neq j, i, j \in\{1,2, \ldots, s\}$.

Simple linear sets (cf. Section 1) of $\mathrm{PG}\left(1, q^{n}\right)$ are exactly those of $\mathrm{CL}$ class one. The next proposition is easy to show.

Proposition 2.6. Let $L_{U}$ be an $\mathbb{F}_{q}$-linear set of $\mathrm{PG}\left(1, q^{n}\right)$ of rank $n$ with maximum field of linearity $\mathbb{F}_{q}$ and let $\varphi$ be a collineation of $\mathrm{PG}\left(1, q^{n}\right)$. Then $L_{U}$ and $L_{U}^{\varphi}$ have the same $\mathcal{Z}(\Gamma \mathrm{L})$-class and $\Gamma \mathrm{L}$-class.

Remark 2.7. Let $L_{U}$ be an $\mathbb{F}_{q}$-linear set of rank $n$ of $\mathrm{PG}\left(1, q^{n}\right)$ with $\Gamma \mathrm{L}$ class $s$ and let $U_{1}, U_{2}, \ldots, U_{s}$ be $\mathbb{F}_{q}$-subspaces belonging to different orbits of $\Gamma \mathrm{L}\left(2, q^{n}\right)$ and defining $L_{U}$. The $\mathrm{P} \Gamma \mathrm{L}\left(2, q^{n}\right)$-orbit of $L_{U}$ is the set

$$
\bigcup_{i=1}^{s}\left\{L_{U_{i}^{f}}: f \in \Gamma L\left(2, q^{n}\right)\right\} .
$$

\section{Examples of simple and non-simple linear sets of $\mathrm{PG}\left(1, q^{n}\right)$}

Let $L_{U}$ be an $\mathbb{F}_{q}$-linear set of rank $n$ of $\operatorname{PG}\left(1, q^{n}\right)$. We can always assume (up to a projectivity) that $L_{U}$ does not contain the point $\langle(0,1)\rangle_{\mathbb{F}_{q^{n}}}$. Then $U=U_{f}=\left\{(x, f(x)): x \in \mathbb{F}_{q^{n}}\right\}$, for some $q$-polynomial $f(x)=\sum_{i=0}^{n-1} a_{i} x^{q^{i}}$ over $\mathbb{F}_{q^{n}}$. For the sake of simplicity we will write $L_{f}$ instead of $L_{U_{f}}$ to denote the linear set defined by $U_{f}$.

According to Result 2.1 and using the same notations as in Section 2.1 if $L_{U}$ is an $\mathbb{F}_{q}$-linear set of rank $n$ of $\mathrm{PG}\left(1, q^{n}\right)$ and $\tau$ is a symplectic polarity, then $U^{\perp^{\prime}}$ defines the same linear set as $U$. Since in general $U^{\perp_{\beta}^{\prime}}$ and $U$ are not equivalent under the action of the group $\Gamma \mathrm{L}\left(2, q^{n}\right)$, simple linear sets of a line are harder to find than non-simple linear sets.

Consider the non-degenerate symmetric bilinear form of $\mathbb{F}_{q^{n}}$ over $\mathbb{F}_{q}$ defined by the following rule

$$
<x, y>:=\operatorname{Tr}_{q^{n} / q}(x y) .
$$

Then the adjoint map $\hat{f}$ of an $\mathbb{F}_{q^{-}}$-linear map $f(x)=\sum_{i=0}^{n-1} a_{i} x^{q^{i}}$ of $\mathbb{F}_{q^{n}}$ (with 
respect to the bilinear form $\langle\rangle$,$) is$

$$
\hat{f}(x):=\sum_{i=0}^{n-1} a_{i}^{q^{n-i}} x^{q^{n-i}} .
$$

Let $\eta: \mathbb{F}_{q^{n}}^{2} \times \mathbb{F}_{q^{n}}^{2} \rightarrow \mathbb{F}_{q^{n}}$ be the non-degenerate alternating bilinear form of $\mathbb{F}_{q^{n}}^{2}$ defined by

$$
\eta((x, y),(u, v))=x v-y u .
$$

Then $\eta$ induces a symplectic polarity on the line $\mathrm{PG}\left(1, q^{n}\right)$ and

$$
\eta^{\prime}((x, y),(u, v))=\operatorname{Tr}_{q^{n} / q}(\eta((x, y),(u, v)))
$$

is a non-degenerate alternating bilinear form on $\mathbb{F}_{q^{n}}^{2}$, when $\mathbb{F}_{q^{n}}^{2}$ is regarded as a $2 n$-dimensional vector space over $\mathbb{F}_{q}$. We will always denote in the paper by $\perp$ and $\perp^{\prime}$ the orthogonal complement maps defined by $\eta$ and $\eta^{\prime}$ on

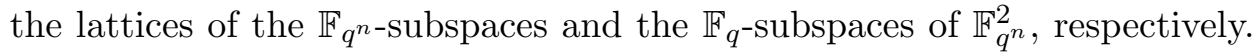
Direct calculation shows that

$$
U_{f}^{\perp^{\prime}}=U_{\hat{f}}
$$

Result 2.1 and (6) allow us to slightly reformulate [4, Lemma 2.6].

Lemma $3.1([4])$. Let $L_{f}=\left\{\langle(x, f(x))\rangle_{\mathbb{F}_{q^{n}}}: x \in \mathbb{F}_{q^{n}}^{*}\right\}$ be an $\mathbb{F}_{q}$-linear set of $\mathrm{PG}\left(1, q^{n}\right)$ of rank $n$, with $f(x)$ a q-polynomial over $\mathbb{F}_{q^{n}}$, and let $\hat{f}$ be the adjoint of $f$ with respect to the bilinear form (2). Then for each point $P \in \mathrm{PG}\left(1, q^{n}\right)$ we have $w_{L_{f}}(P)=w_{L_{\hat{f}}}(P)$. In particular, $L_{f}=L_{\hat{f}}$ and the maps defined by $f(x) / x$ and $\hat{f}(x) / x$ have the same image.

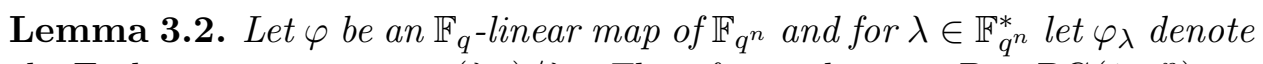
the $\mathbb{F}_{q}$-linear map: $x \mapsto \varphi(\lambda x) / \lambda$. Then for each point $P \in \operatorname{PG}\left(1, q^{n}\right)$ we have $w_{L_{\varphi}}(P)=w_{L_{\varphi_{\lambda}}}(P)$. In particular, $L_{\varphi}=L_{\varphi_{\lambda}}$.

Proof. The statements follow from $\lambda U_{\varphi_{\lambda}}=U_{\varphi}$.

Remark 3.3. The results of Lemmas 3.1 and 3.2 can also be obtained via Dickson matrices. For a q-polynomial $f(x)=\sum_{i=0}^{n-1} a_{i} x^{q^{i}}$ over $\mathbb{F}_{q^{n}}$ let $D_{f}$ denote the associated Dickson matrix (or q-circulant matrix)

$$
D_{f}:=\left(\begin{array}{cccc}
a_{0} & a_{1} & \ldots & a_{n-1} \\
a_{n-1}^{q} & a_{0}^{q} & \ldots & a_{n-2}^{q} \\
\vdots & \vdots & \vdots & \vdots \\
a_{1}^{q^{n-1}} & a_{2}^{q^{n-1}} & \ldots & a_{0}^{q^{n-1}}
\end{array}\right)
$$


When $f(x)=\lambda x$ for some $\lambda \in \mathbb{F}_{q^{n}}$ we will simply write $D_{\lambda}$. The rank of the matrix $D_{f}$ equals the rank of the $\mathbb{F}_{q}$-linear map $f$, see for example [29]. We will denote the point $\langle(1, \lambda)\rangle_{q^{n}}$ by $P_{\lambda}$.

Transposition preserves the rank of matrices and $D_{f}^{T}=D_{\hat{f}}, D_{\lambda}^{T}=D_{\lambda}$. It follows that

$$
\operatorname{dim}_{q} \operatorname{ker}\left(D_{f}-D_{\lambda}\right)=\operatorname{dim}_{q} \operatorname{ker}\left(D_{f}-D_{\lambda}\right)^{T}=\operatorname{dim}_{q} \operatorname{ker}\left(D_{\hat{f}}-D_{\lambda}\right),
$$

and hence for each $\lambda \in \mathbb{F}_{q^{n}}$ we have $w_{L_{f}}\left(P_{\lambda}\right)=w_{L_{\hat{f}}}\left(P_{\lambda}\right)$.

Let $f_{\mu}(x)=f(x \mu) / \mu$. It is easy to see that $D_{1 / \mu} D_{f} D_{\mu}=D_{f_{\mu}}$ and

$$
\operatorname{dim}_{q} \operatorname{ker}\left(D_{f}-D_{\lambda}\right)=\operatorname{dim}_{q} \operatorname{ker} D_{1 / \mu}\left(D_{f}-D_{\lambda}\right) D_{\mu}=\operatorname{dim}_{q} \operatorname{ker}\left(D_{f_{\mu}}-D_{\lambda}\right),
$$

and hence $w_{L_{f}}\left(P_{\lambda}\right)=w_{L_{f_{\mu}}}\left(P_{\lambda}\right)$ for each $\lambda \in \mathbb{F}_{q^{n}}$.

From the previous arguments it follows that linear sets $L_{f}$ with $f(x)=$ $\hat{f}(x)$ are good candidates for being simple. In the next section we show that the trace function, which has the previous property, defines a simple linear set. We are going to use the following lemmas which will also be useful later.

Lemma 3.4. Let $f$ and $g$ be two linearized polynomials. If $L_{f}=L_{g}$, then for each positive integer $d$ the following holds

$$
\sum_{x \in \mathbb{F}_{q^{n}}^{*}}\left(\frac{f(x)}{x}\right)^{d}=\sum_{x \in \mathbb{F}_{q^{n}}^{*}}\left(\frac{g(x)}{x}\right)^{d} .
$$

Proof. If $L_{f}=L_{g}=: L$, then $\left\{f(x) / x: x \in \mathbb{F}_{q^{n}}^{*}\right\}=\left\{g(x) / x: x \in \mathbb{F}_{q^{n}}^{*}\right\}=: H$. For each $h \in H$ we have $|\{x: f(x) / x=h\}|=q^{i}-1$, where $i$ is the weight of the point $\langle(1, h)\rangle_{q^{n}} \in L$ w.r.t. $U_{f}$, and similarly $|\{x: g(x) / x=h\}|=q^{j}-1$, where $j$ is the weight of the point $\langle(1, h)\rangle_{q^{n}} \in L$ w.r.t. $U_{g}$. Because of the characteristic of $\mathbb{F}_{q^{n}}$, we obtain:

$$
\sum_{x \in \mathbb{F}_{q^{n}}^{*}}\left(\frac{f(x)}{x}\right)^{d}=-\sum_{h \in H} h^{d}=\sum_{x \in F_{q^{n}}^{*}}\left(\frac{g(x)}{x}\right)^{d} .
$$

For the sake of completeness we give a proof of the following well-known result.

Lemma 3.5. For any prime power $q$ and integer $d$ we have $\sum_{x \in \mathbb{F}_{q}^{*}} x^{d}=-1$ if $q-1 \mid d$ and $\sum_{x \in \mathbb{F}_{q}^{*}} x^{d}=0$ otherwise. 
Proof. Let $g$ denote a primitive element of $\mathbb{F}_{q}$ and put $s=\sum_{j=0}^{n-2} g^{i d}$. Then $s g^{d}=s$ and hence either $s=0$, or $g^{d}=1$. In the latter case $q-1 \mid d$ since $g$ was a primitive element and hence $x^{d}=1$ for each $x \in \mathbb{F}_{q}^{*}$.

Lemma 3.6. Let $f(x)=\sum_{i=0}^{n-1} a_{i} x^{q^{i}}$ and $g(x)=\sum_{i=0}^{n-1} b_{i} x^{q^{i}}$ be two qpolynomials over $\mathbb{F}_{q^{n}}$, such that $L_{f}=L_{g}$. Then

$$
a_{0}=b_{0},
$$

and for $k=1,2, \ldots, n-1$ it holds that

$$
a_{k} a_{n-k}^{q^{k}}=b_{k} b_{n-k}^{q^{k}},
$$

for $k=2,3, \ldots, n-1$ it holds that

$$
a_{1} a_{k-1}^{q} a_{n-k}^{q^{k}}+a_{k} a_{n-1}^{q} a_{n-k+1}^{q^{k}}=b_{1} b_{k-1}^{q} b_{n-k}^{q^{k}}+b_{k} b_{n-1}^{q} b_{n-k+1}^{q^{k}} .
$$

Proof. We are going to use Lemma 3.5 together with Lemma 3.4 with different choices of $d$.

With $d=1$ we have

$$
\sum_{x \in \mathbb{F}_{q^{n}}^{*}} \sum_{i=0}^{n-1} a_{i} x^{q^{i}-1}=\sum_{x \in \mathbb{F}_{q^{*}}^{*}} \sum_{i=0}^{n-1} b_{i} x^{q^{i}-1}
$$

and hence

$$
\sum_{i=0}^{n-1} a_{i} \sum_{x \in \mathbb{F}_{q^{n}}^{*}} x^{q^{i}-1}=\sum_{i=0}^{n-1} b_{i} \sum_{x \in \mathbb{F}_{q^{*}}^{*}} x^{q^{i}-1} .
$$

Since $q^{n}-1$ cannot divide $q^{i}-1$ with $i=1,2, \ldots, n-1, a_{0}=b_{0}=: c$ follows. Let $\varphi$ denote the $\mathbb{F}_{q^{n}}$-linear map which fixes $(0,1)$ and maps $(1,0)$ to $(1,-c)$. Then $U_{f}^{\varphi}=U_{f^{\prime}}$ and $U_{g}^{\varphi}=U_{g^{\prime}}$ with $f^{\prime}=\sum_{i=1}^{n-1} a_{i} x^{q^{i}}, g^{\prime}=\sum_{i=1}^{n-1} b_{i} x^{q^{i}}$ and of course with $L_{f^{\prime}}=L_{g^{\prime}}$. It follows that we may assume $c=0$.

First we show that (8) holds. With $d=q^{k}+1,1 \leq k \leq n-1$ we obtain

$$
\sum_{1 \leq i, j \leq n-1} a_{i} a_{j}^{q^{k}} \sum_{x \in \mathbb{F}_{q^{*}}^{*}} x^{q^{i}-1+q^{j+k}-q^{k}}=\sum_{1 \leq i, j \leq n-1} b_{i} b_{j}^{q^{k}} \sum_{x \in \mathbb{F}_{q^{*}}^{*}} x^{q^{i}-1+q^{j+k}-q^{k}} .
$$

$\sum_{x \in \mathbb{F}_{q^{n}}^{*}} x^{q^{i}-1+q^{j+k}-q^{k}}=-1$ if and only if $q^{i}+q^{j+k} \equiv q^{k}+1\left(\bmod q^{n}-1\right)$, and zero otherwise. Suppose that the former case holds.

First consider $j+k \leq n-1$. Then $q^{i}+q^{j+k} \leq q^{n-1}+q^{n-1}<q^{k}+1+$ $2\left(q^{n}-1\right)$ hence one of the following holds. 
- If $q^{i}+q^{j+k}=q^{k}+1$, then the right hand side is not divisible by $q$, a contradiction.

- If $q^{i}+q^{j+k}=q^{k}+1+\left(q^{n}-1\right)=q^{n}+q^{k}$, then $j+k=n$, a contradiction.

Now consider the case $j+k \geq n$. Then $q^{i}+q^{j+k} \equiv q^{i}+q^{j+k-n} \equiv q^{k}+1$ $\left(\bmod q^{n}-1\right)$. Since $j+k \leq 2(n-1)$, we have $q^{i}+q^{j+k-n} \leq q^{n-1}+q^{n-2}<$ $q^{k}+1+2\left(q^{n}-1\right)$, hence one of the following holds.

- If $q^{i}+q^{j+k-n}=q^{k}+1$, then $j+k=n$ and $i=k$.

- If $q^{i}+q^{j+k-n}=q^{k}+1+\left(q^{n}-1\right)=q^{n}+q^{k}$, then there is no solution since $j+k-n \notin\{k, n\}$.

Hence (8) follows. Now we show that (9) also holds. Note that in this case $n \geq 3$, otherwise there is no $k$ with $2 \leq k \leq n-1$. With $d=q^{k}+q+1$, we obtain

$$
\begin{gathered}
\sum_{1 \leq i, j, m \leq n-1} a_{i} a_{j}^{q} a_{m}^{q^{k}} \sum_{x \in \mathbb{F}_{q^{*}}^{*}} x^{q^{i}-1+q^{j+1}-q+q^{m+k}-q^{k}}= \\
\sum_{1 \leq i, j, m \leq n-1} b_{i} b_{j}^{q} b_{m}^{q^{k}} \sum_{x \in \mathbb{F}_{q^{*}}^{*}} x^{q^{i}-1+q^{j+1}-q+q^{m+k}-q^{k}} .
\end{gathered}
$$

$\sum_{x \in \mathbb{F}_{q^{*}}^{*}} x^{q^{i}-1+q^{j+1}-q+q^{m+k}-q^{k}}=-1$ if and only if $q^{i}+q^{j+1}+q^{m+k} \equiv q^{k}+q+1$ $\left(\bmod q^{n}-1\right)$, and zero otherwise. Suppose that the former case holds.

First consider $m+k \leq n-1$. Then $q^{i}+q^{j+1}+q^{m+k} \leq q^{n-1}+q^{n}+q^{n-1}<$ $q^{k}+q+1+2\left(q^{n}-1\right)$ hence one of the following holds.

- If $q^{i}+q^{j+1}+q^{m+k}=q^{k}+q+1$, then the right hand side is not divisible by $q$, a contradiction.

- If $q^{i}+q^{j+1}+q^{m+k}=q^{k}+q+1+\left(q^{n}-1\right)=q^{n}+q^{k}+q$, then $m+k=n$, $j+1=k$ and $i=1$, a contradiction.

Now consider the case $m+k \geq n$. Then $q^{i}+q^{j+1}+q^{m+k} \equiv q^{i}+$ $q^{j+1}+q^{m+k-n} \equiv q^{k}+q+1\left(\bmod q^{n}-1\right)$. We have $q^{i}+q^{j+1}+q^{m+k-n} \leq$ $q^{n-1}+q^{n}+q^{n-2}<q^{k}+q+1+2\left(q^{n}-1\right)$ hence one of the following holds.

- If $q^{i}+q^{j+1}+q^{m+k-n}=q^{k}+q+1$, then $j+1=k, i=1$ and $m+k=n$.

- If $q^{i}+q^{j+1}+q^{m+k-n}=q^{k}+q+1+\left(q^{n}-1\right)=q^{n}+q^{k}+q$, then $j+1=n, i=k$ and $m+k=n+1$.

This concludes the proof. 


\subsection{Linear sets defined by the trace function}

We show that there exists at least one simple $\mathbb{F}_{q}$-linear set in $\operatorname{PG}\left(1, q^{n}\right)$ for each $q$ and $n$. Let $V=\left\{\left(x, \operatorname{Tr}_{q^{n} / q}(x)\right): x \in \mathbb{F}_{q^{n}}\right\}$. We show that $L_{U}=L_{V}$

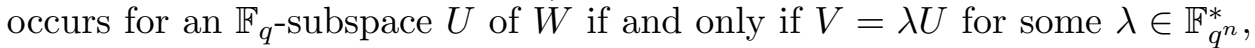
i.e. $L_{V}$ is of $\mathcal{Z}(\Gamma L)$-class one and hence simple.

Theorem 3.7. Let $V=\left\{\left(x, \operatorname{Tr}_{q^{n} / q}(x)\right): x \in \mathbb{F}_{q^{n}}\right\}$, then the $\mathbb{F}_{q^{-}}$linear set $L_{V}$ of $\mathrm{PG}\left(1, q^{n}\right)$ is of $\mathcal{Z}(\Gamma \mathrm{L})$-class one.

Proof. Suppose $L_{U_{f}}=L_{V}$ with $U_{f}=\left\{(x, f(x)): x \in \mathbb{F}_{q^{n}}\right\}$ and $f(x)=$ $\sum_{i=0}^{n-1} a_{i} x^{q^{i}}$. We are going to use Lemma 3.6 with $g(x)=\operatorname{Tr}_{q^{n} / q}(x)$. The coefficients $b_{0}, b_{1}, \ldots, b_{n-1}$ of $g(x)$ are 1 , hence $a_{0}=1$, and for $k=1,2, \ldots, n-$ 1

$$
a_{k} a_{n-k}^{q^{k}}=1
$$

for $k=2,3, \ldots, n-1$

$$
a_{1} a_{k-1}^{q} a_{n-k}^{q^{k}}+a_{k} a_{n-1}^{q} a_{n-k+1}^{q^{k}}=2 .
$$

Note that 10 implies $a_{i} \neq 0$ for $i=1,2, \ldots, n-1$. First we prove

$$
a_{i}=a_{1}^{1+q+\ldots+q^{i-1}}
$$

by induction on $i$ for each $0<i<n$. The assertion holds for $i=1$. Suppose that it holds for some integer $i-1$ with $1<i<n$. We prove that it also holds for $i$. Then (11) with $k=i$ gives

$$
a_{1} a_{i-1}^{q} a_{n-i}^{q^{i}}+a_{i} a_{n-1}^{q} a_{n-i+1}^{q^{i}}=2 .
$$

Also, 10 with $k=i, k=i-1$ and $k=1$, respectively, gives

$$
\begin{gathered}
a_{n-i}^{q^{i}}=1 / a_{i}, \\
a_{n-i+1}^{q^{i}}=1 / a_{i-1}^{q}, \\
a_{n-1}^{q}=1 / a_{1} .
\end{gathered}
$$

Then (13) gives

$$
a_{1} a_{i-1}^{q} / a_{i}+a_{i} /\left(a_{1} a_{i-1}^{q}\right)=2 .
$$

It follows that $a_{1} a_{i-1}^{q} / a_{i}=1$ and hence the induction hypothesis on $a_{i-1}$ yields $a_{i}=a_{1}^{1+q+\ldots+q^{i-1}}$. 
Finally we show $\mathrm{N}\left(a_{1}\right)=1$. First consider $n$ even. Then (10) with $k=n / 2$ gives $a_{n / 2}^{q^{n / 2}+1}=1$. Applying 12 yields $\mathrm{N}\left(a_{1}\right)=1$. If $n$ is odd, then 10 with $k=(n-1) / 2$ gives $a_{(n-1) / 2} a_{(n+1) / 2}^{q^{(n-1) / 2}}=1$. Applying 12 yields $\mathrm{N}\left(a_{1}\right)=1$. It follows that $a_{1}=\lambda^{q-1}$ for some $\lambda \in \mathbb{F}_{q^{n}}^{*}$ and hence $f(x)=\sum_{i=0}^{n-1} \lambda^{q^{i}-1} x^{q^{i}}$. Then $\lambda U_{f}=\left\{\left(x, \operatorname{Tr}_{q^{n} / q}(x)\right): x \in \mathbb{F}_{q^{n}}^{*}\right\}$.

Remark 3.8. We point out that in the above theorem we do not have any assumption on the weight of points of $L_{U}$. In the special case when $L_{U}=L_{V}$ and $L_{U}$ has a point of weight $n-1$, then the $\mathrm{GL}\left(2, q^{n}\right)$-equivalence of $U$ and $V$ can be deduced also from [8, Theorem 2.3].

\subsection{Non-simple linear sets}

An $\mathbb{F}_{q}$-linear set of pseudoregulus type of $\mathrm{PG}\left(1, q^{n}\right)$ is any linear set equivalent to $\left\{\left\langle\left(x, x^{q}\right)\right\rangle_{\mathbb{F}_{q^{n}}}: x \in \mathbb{F}_{q^{n}}^{*}\right\}$. In [7] it was proved that the $\Gamma L$-class of such linear sets is $\varphi(n) / 2$, hence they are non-simple for $n=5$ and $n>6$. So far, these are the only known non-simple linear sets of $\operatorname{PG}\left(1, q^{n}\right)$. Here we show that $\mathbb{F}_{q}$-linear sets $L_{f}$ of $\mathrm{PG}\left(1, q^{n}\right)$ introduced by Lunardon and Polverino, which are not of pseudoregulus type ([23, Theorems 2 and 3]), are non-simple as well. Let us start by proving the following preliminary result.

Proposition 3.9. Let $f(x)=\sum_{i=0}^{n-1} a_{i} x^{q^{i}}$. There is an $\mathbb{F}_{q^{n} \text {-semilinear map }}$ between $U_{f}$ and $U_{\hat{f}}$ if and only if the following system of $n$ equations has a solution $A, B, C, D \in \mathbb{F}_{q^{n}}, A D-B C \neq 0, \sigma=p^{k}$ :

$$
\begin{gathered}
C+D a_{0}^{\sigma}-a_{0} A=\sum_{i=0}^{n-1}\left(B a_{i} a_{i}^{\sigma}\right)^{q^{n-i}}, \\
\ldots \\
D a_{m}^{\sigma}-\left(a_{n-m} A\right)^{q^{m}}=\sum_{i=0}^{n-1}\left(B a_{i} a_{i+m}^{\sigma}\right)^{q^{n-i}}, \text { with } m=1, \ldots, n-2, \\
\ldots \\
D a_{n-1}^{\sigma}-\left(a_{1} A\right)^{q^{n-1}}=\sum_{i=0}^{n-1}\left(B a_{i} a_{i+n-1}^{\sigma}\right)^{q^{n-i}},
\end{gathered}
$$

where the indices are taken modulo $n$. 
Proof. Because of cardinality reasons the condition $A D-B C \neq 0$ is necessary. Then

$$
\left\{\left(\begin{array}{c}
x \\
\hat{f}(x)
\end{array}\right): x \in \mathbb{F}_{q^{n}}\right\}=\left\{\left(\begin{array}{cc}
A & B \\
C & D
\end{array}\right)\left(\begin{array}{c}
x^{\sigma} \\
f(x)^{\sigma}
\end{array}\right): x \in \mathbb{F}_{q^{n}}\right\}
$$

holds if and only if

$$
C x^{\sigma}+D \sum_{j=0}^{n-1} a_{j}^{\sigma} x^{\sigma q^{j}}=\sum_{i=0}^{n-1} a_{n-i}^{q^{i}}\left(A x^{\sigma}+B \sum_{j=0}^{n-1} a_{j}^{\sigma} x^{\sigma q^{j}}\right)^{q^{i}}
$$

for each $x \in \mathbb{F}_{q^{n}}$. After reducing modulo $x^{q^{n}}-x$, this is a polynomial equation of degree at most $q^{n-1}$ in the variable $x^{\sigma}$. It follows that it holds for each $x \in \mathbb{F}_{q^{n}}$ if and only if it is the zero polynomial. Comparing coefficients on both sides yields the assertion.

We are able to prove the following.

Proposition 3.10. Consider a polynomial of the form $f(x)=\delta x^{q}+x^{q^{n-1}}$, where $q>4$ is a power of the prime $p$. If $n>4$, then for each generator $\delta$ of the multiplicative group of $\mathbb{F}_{q^{n}}$ the linear set $L_{f}$ is not simple.

Proof. Lemma 3.1 yields $L_{f}=L_{\hat{f}}$ thus it is enough to show the existence

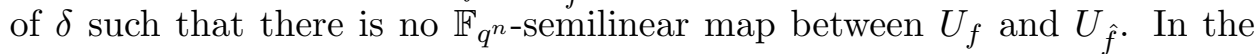
equations of Proposition 3.9 we have $a_{1}=\delta, a_{n-1}=1$ and $a_{0}=a_{2}=$ $\ldots=a_{n-2}=0$. If $n>4$ then the first two and the last two equations of Proposition 3.9 give

$$
\begin{gathered}
C=\left(B \delta^{\sigma+1}\right)^{q^{n-1}}+B^{q}, \\
D \delta^{\sigma}-A^{q}=0, \\
0=(B \delta)^{q^{n-1}}, \\
D-(\delta A)^{q^{n-1}}=0,
\end{gathered}
$$

where $\sigma=p^{k}$ for some integer $k$. If there is a solution, then $B=C=0$ and $(\delta A)^{q^{n-1}} \delta^{\sigma}=A^{q}$. Taking $q$-th powers on both sides yield

$$
\delta^{\sigma q+1}=A^{q^{2}-1}
$$

and hence

$$
\delta^{\frac{(\sigma q+1)\left(q^{n}-1\right)}{q-1}}=1
$$


For each $\sigma$ let $G_{\sigma}$ be the set of elements $\delta$ of $\mathbb{F}_{q^{n}}$ satisfying (16). For each $\sigma$, $G_{\sigma}$ is a subgroup of the multiplicative group $M$ of $\mathbb{F}_{q^{n}}$. We show that these are proper subgroups of $M$. We have $G_{p^{k}}=M$ if and only if $q^{n}-1$ divides $\frac{\left(p^{k} q+1\right)\left(q^{n}-1\right)}{q-1}$, i.e. when $q-1$ divides $p^{k} q+1$. Since $\operatorname{gcd}\left(p^{w}+1, p^{v}-1\right)$ is always 1,2 , or $p^{\operatorname{gcd}(w, v)}+1$, it follows that for $q>4$ we cannot have $q-1$ as a divisor of $p^{k} q+1$.

It follows that for any generator $\delta$ of $M$ we have $\delta \notin \cup_{j} G_{p^{j}}$ and hence $\delta^{\sigma q+1} \neq A^{q^{2}-1}$ for each $\sigma$ and for each $A$.

Remark 3.11. If $q=4$, then (15) with $k=2(n-1)+1$ asks for the solution of $\delta^{3}=A^{15}$. When $n$ is odd, then $\left\{x^{3}: x \in \mathbb{F}_{4^{n}}\right\}=\left\{x^{15}: x \in \mathbb{F}_{4^{n}}\right\}$ and hence for each $\delta$ there exists $A$ such that $\delta^{3}=A^{15}$.

If $q=3$, then (15) with $k=n-1$ asks for the solution of $\delta^{2}=A^{8}$. When $n$ is odd, then $\left\{x^{2}: x \in \mathbb{F}_{3^{n}}\right\}=\left\{x^{8}: x \in \mathbb{F}_{3^{n}}\right\}$ and hence for each $\delta$ there exists $A$ such that $\delta^{2}=A^{8}$.

If $q=2$, then (15) with $k=0$ asks for the solution of $\delta^{3}=A^{3}$. This equation always has a solution.

\section{Linear sets of rank 4 of $\mathrm{PG}\left(1, q^{4}\right)$}

$\mathbb{F}_{q}$-linear sets of rank two of $\mathrm{PG}\left(1, q^{2}\right)$ are the Baer sublines, which are equivalent. As we have mentioned in the introduction, subgeometries are simple linear sets, in fact they have $\mathcal{Z}(\Gamma \mathrm{L})$-class one (cf. [19, Theorem 2.6] and [15, Section 25.5]). There are two non-equivalent $\mathbb{F}_{q}$-linear sets of rank 3 of $\mathrm{PG}\left(1, q^{3}\right)$, the linear sets of size $q^{2}+q+1$ and those of size $q^{2}+1$. Linear sets in both families are equivalent, since the stabilizer of a $q$-order subgeometry $\Sigma$ of $\Sigma^{*}=\operatorname{PG}\left(2, q^{3}\right)$ is transitive on the set of those points of $\Sigma^{*} \backslash \Sigma$ which are incident with a line of $\Sigma$ and on the set of points of $\Sigma^{*}$ not incident with any line of $\Sigma$ (cf. Section 5.2 and [18]). In the first case we have the linear sets of pseudoregulus type with $\Gamma$-class 1 and $\mathcal{Z}(\Gamma L)$-class 2 (cf. Remark 5.6 and Example 5.1). In the second case we have the linear sets defined by $\operatorname{Tr}_{q^{3} / q}$ with $\Gamma$ L-class and $\mathcal{Z}(\Gamma L)$-class 1 (cf. Theorem 3.7, see also [12, Corollary 6]).

From [6, Proposition 2.3] it follows that $\mathbb{F}_{q}$-linear sets of rank 5 in $\mathrm{PG}\left(W, q^{4}\right)=\mathrm{PG}\left(2, q^{4}\right)$ are simple. The orbits of 5 -dimensional $\mathbb{F}_{q}$-subspaces of $W$ under $\Gamma \mathrm{L}\left(3, q^{4}\right)$ are also determined (cf. [6, pg. 54]). The results related to Rédei type blocking sets allow to determine all the orbits of 4dimensional $\mathbb{F}_{q^{-}}$-subspaces of a two-dimensional $\mathbb{F}_{q^{4}}$-space under the group $\Gamma \mathrm{L}\left(2, q^{4}\right)$. The aim of this section is to prove that $\mathbb{F}_{q}$-linear sets of rank 4 in 
$\mathrm{PG}\left(1, q^{4}\right)$, with maximum field of linearity $\mathbb{F}_{q}$, are simple (cf. Theorem 4.5), since this does not follow from the above mentioned simplicity of $\mathbb{F}_{q}$-linear blocking sets. As a corollary, a list of orbits under $\operatorname{P\Gamma L}\left(2, q^{4}\right)$ of $\mathbb{F}_{q^{-}}$-linear sets of rank 4 in $\mathrm{PG}\left(1, q^{4}\right)$ can be deduced from [6, pg. 54].

\subsection{Subspaces defining the same linear set}

Lemma 4.1. Let $f(x)=\sum_{i=0}^{3} a_{i} x^{q^{i}}$ and $g(x)=\sum_{i=0}^{3} b_{i} x^{q^{i}}$ be two $q$ polynomials over $\mathbb{F}_{q^{4}}$, such that $L_{f}=L_{g}$. Then

$$
\begin{gathered}
\mathrm{N}\left(a_{1}\right)+\mathrm{N}\left(a_{2}\right)+\mathrm{N}\left(a_{3}\right)+a_{1}^{1+q^{2}} a_{3}^{q+q^{3}}+a_{1}^{q+q^{3}} a_{3}^{1+q^{2}}+\operatorname{Tr} q^{4} / q\left(a_{1} a_{2}^{q+q^{2}} a_{3}^{q^{3}}\right)= \\
\mathrm{N}\left(b_{1}\right)+\mathrm{N}\left(b_{2}\right)+\mathrm{N}\left(b_{3}\right)+b_{1}^{1+q^{2}} b_{3}^{q+q^{3}}+b_{1}^{q+q^{3}} b_{3}^{1+q^{2}}+\operatorname{Tr} q^{4} / q\left(b_{1} b_{2}^{q+q^{2}} b_{3}^{q^{3}}\right) .
\end{gathered}
$$

Proof. We are going to follow the proof of Lemma 3.6. As in that proof, we may assume $a_{0}=b_{0}=0$. In Lemma 3.4 take $d=1+q+q^{2}+q^{3}$. We obtain

$$
\begin{aligned}
& \sum_{1 \leq i, j, k, m \leq 3} a_{i} a_{j}^{q} a_{k}^{q^{2}} a_{m}^{q^{3}} \sum_{x \in \mathbb{F}_{q^{*}}^{*}} x^{q^{i}-1+q^{j+1}-q+q^{k+2}-q^{2}+q^{m+3}-q^{3}}= \\
& \sum_{1 \leq i, j, k, m \leq 3} b_{i} b_{j}^{q} b_{k}^{q^{2}} b_{m}^{q^{3}} \sum_{x \in \mathbb{F}_{q^{*}}^{*}} x^{q^{i}-1+q^{j+1}-q+q^{k+2}-q^{2}+q^{m+3}-q^{3}} . \\
& \sum_{x \in \mathbb{F}_{q^{4}}^{*}} x^{q^{i}-1+q^{j+1}-q+q^{k+2}-q^{2}+q^{m+3}-q^{3}}=-1 \text { if and only if } \\
& q^{i}+q^{j+1}+q^{k+2}+q^{m+3} \equiv q^{i}+q^{j+1}+q^{k+2}+q^{m-1} \equiv 1+q+q^{2}+q^{3} \quad\left(\bmod q^{4}-1\right),
\end{aligned}
$$

and zero otherwise. Suppose that the former case holds.

First consider $k=1$. Then $q^{i}+q^{j+1}+q^{k+2}+q^{m-1} \leq q^{3}+q^{4}+q^{3}+q^{2}<$ $1+q+q^{2}+q^{3}+2\left(q^{4}-1\right)$ hence one of the following holds.

- If $q^{i}+q^{j+1}+q^{k+2}+q^{m-1}=1+q+q^{2}+q^{3}$, then $m=i=j=k=1$.

- If $q^{i}+q^{j+1}+q^{k+2}+q^{m-1}=1+q+q^{2}+q^{3}+q^{4}-1=q+q^{2}+q^{3}+q^{4}$, then $\{i, j+1, k+2, m-1\}=\{1,2,3,4\}$, hence one of the following holds

$$
\begin{aligned}
& i=1, j=3, k=1, m=3, \\
& i=2, j=3, k=1, m=2 .
\end{aligned}
$$

Now consider the case $k \geq 2$. Then $q^{i}+q^{j+1}+q^{k+2}+q^{m-1} \equiv q^{i}+q^{j+1}+$ $q^{k-2}+q^{m-1} \leq q^{3}+q^{4}+q+q^{2}<1+q+q^{2}+q^{3}+2\left(q^{4}-1\right)$ hence one of the following holds. 
- If $q^{i}+q^{j+1}+q^{k-2}+q^{m-1}=1+q+q^{2}+q^{3}$, then $\{i, j+1, k-2, m-1\}=$ $\{0,1,2,3\}$, hence one of the following holds

$$
\begin{aligned}
& i=1, j=2, k=2, m=3, \\
& i=2, j=2, k=2, m=2, \\
& i=2, j=2, k=3, m=1, \\
& i=3, j=1, k=2, m=2, \\
& i=3, j=1, k=3, m=1 .
\end{aligned}
$$

- If $q^{i}+q^{j+1}+q^{k-2}+q^{m-1}=1+q+q^{2}+q^{3}+q^{4}-1=q+q^{2}+q^{3}+q^{4}$, then $i=j=k=m=3$.

Proposition 4.2. Let $f(x)$ and $g(x)$ be two q-polynomials over $\mathbb{F}_{q^{4}}$ such that $L_{f}=L_{g}$. If the maximum field of linearity of $f$ is $\mathbb{F}_{q}$, then

$$
g(x)=f(\lambda x) / \lambda
$$

or

$$
g(x)=\hat{f}(\lambda x) / \lambda .
$$

Proof. By Proposition 2.3, the maximum field of linearity of $g$ is also $\mathbb{F}_{q}$. First note that $L_{g}=L_{f}$ when $g$ is as in the assertion (cf. Lemmas 3.1 and 3.2. . Let $f(x)=\sum_{i=0}^{3} a_{i} x^{q^{i}}$ and $g(x)=\sum_{i=0}^{3} b_{i} x^{q^{i}}$.

First we are going to use Lemma 3.6. From (7) we have $a_{0}=b_{0}$. From (8) with $n=4$ and $k=1,2$ we have $a_{1} a_{3}^{q}=b_{1} b_{3}^{q}$ and $a_{2}^{1+q^{2}}=b_{2}^{1+q^{2}}$, respectively. From (9) with $n=4$ and $k=2$ we obtain

$$
a_{1}^{q+1} a_{2}^{q^{2}}+a_{2} a_{3}^{q+q^{2}}=b_{1}^{q+1} b_{2}^{q^{2}}+b_{2} b_{3}^{q+q^{2}} .
$$

Note that $a_{1} a_{3}^{q}=b_{1} b_{3}^{q}$ implies

$$
\mathrm{N}\left(b_{1}\right) \mathrm{N}\left(b_{3}\right)=\mathrm{N}\left(a_{1}\right) \mathrm{N}\left(a_{3}\right) .
$$

Multiplying (17) by $b_{2}$ and applying $a_{2}^{1+q^{2}}=b_{2}^{1+q^{2}}$ yields:

$$
b_{2}^{2} b_{3}^{q^{2}+q}-b_{2}\left(a_{1}^{q+1} a_{2}^{q^{2}}+a_{2} a_{3}^{q^{2}+q}\right)+b_{1}^{q+1} a_{2}^{q^{2}+1}=0 .
$$


First suppose $b_{1} b_{2} b_{3} \neq 0$. Then $(19)$ is a second degree polynomial in $b_{2}$. Applying $a_{1} a_{3}^{q}=b_{1} b_{3}^{q}$ it is easy to see that the roots of (19) are

$$
\begin{aligned}
& b_{2,1}=\frac{a_{1}^{q+1} a_{2}^{q^{2}}}{b_{3}^{q^{2}+q}}, \\
& b_{2,2}=\frac{a_{2} a_{3}^{q^{2}+q}}{b_{3}^{q^{2}+q}} .
\end{aligned}
$$

First we consider $b_{2}=b_{2,1}$. Then $a_{2}^{1+q^{2}}=b_{2}^{1+q^{2}}$ yields $\mathrm{N}\left(a_{1}\right)=\mathrm{N}\left(b_{3}\right)$ and hence $\mathrm{N}\left(b_{1}\right)=\mathrm{N}\left(a_{3}\right)$. In particular, $\mathrm{N}\left(b_{1} / a_{3}^{q}\right)=1$ and hence $b_{1}=a_{3}^{q} \lambda^{q-1}$ for some $\lambda \in \mathbb{F}_{q^{4}}^{*}$. From $a_{1} a_{3}^{q}=b_{1} b_{3}^{q}$ we obtain $b_{3}=a_{1}^{q^{3}} a_{3} / b_{1}^{q^{3}}=a_{1}^{q^{3}} \lambda^{q^{3}-1}$. Applying this we get $b_{2}=a_{1}^{q+1} a_{2}^{q^{2}} / b_{3}^{q^{2}+q}=a_{2}^{q^{2}} \lambda^{q^{2}-1}$ and hence

$$
g(x)=a_{0} x+a_{3}^{q} \lambda^{q-1} x^{q}+a_{2}^{q^{2}} \lambda^{q^{2}-1} x^{q^{2}}+a_{1}^{q^{3}} \lambda^{q^{3}-1} x^{q^{3}}=f(\lambda x) / \lambda .
$$

as we claimed.

Now consider $b_{2}=b_{2,2}$. Then $a_{2}^{1+q^{2}}=b_{2}^{1+q^{2}}$ yields $\mathrm{N}\left(a_{3}\right)=\mathrm{N}\left(b_{3}\right)$ and hence $\mathrm{N}\left(a_{1}\right)=\mathrm{N}\left(b_{1}\right)$. Hence $b_{1}=a_{1} \lambda^{q-1}$ for some $\lambda \in \mathbb{F}_{q^{4}}^{*}$. From $a_{1} a_{3}^{q}=b_{1} b_{3}^{q}$ we obtain $b_{3}=a_{1}^{q^{3}} a_{3} / b_{1}^{q^{3}}=a_{3} \lambda^{q^{3}-1}$. Applying this we obtain $b_{2}=a_{2} a_{3}^{q^{2}+q} / b_{3}^{q^{2}+q}=a_{2} \lambda^{q^{2}-1}$ and hence

$$
g(x)=a_{0} x+a_{1} \lambda^{q-1} x^{q}+a_{2}^{q^{2}} \lambda^{q^{2}-1} x^{q^{2}}+a_{3}^{q^{3}} \lambda^{q^{3}-1} x^{q^{3}}=\hat{f}(\lambda x) / \lambda .
$$

If $b_{1}=b_{3}=0$, then either $b_{2}=0$ and the maximum field of linearity of $g(x)$ is $\mathbb{F}_{q^{4}}$, or $b_{2} \neq 0$ and the maximum field of linearity of $g(x)$ is $\mathbb{F}_{q^{2}}$. Thus we may assume $b_{1} \neq 0$ or $b_{3} \neq 0$.

First assume $b_{2} \neq 0$ and $b_{1}=0$. Then $b_{3} \neq 0$ and $(19)$ gives

$$
b_{2} b_{3}^{q^{2}+q}=a_{1}^{q+1} a_{2}^{q^{2}}+a_{2} a_{3}^{q^{2}+q} .
$$

Then $a_{1} a_{3}^{q}=b_{1} b_{3}^{q}$ yields either $a_{1}=0$ and $b_{2} b_{3}^{q^{2}+q}=a_{2} a_{3}^{q^{2}+q}$, or $a_{3}=0$ and $b_{2} b_{3}^{q^{2}+q}=a_{1}^{q+1} a_{2}^{q^{2}}$. Taking $\left(q^{2}+1\right)$-powers on both sides gives $b_{2}^{q^{2}+1} \mathrm{~N}\left(b_{3}\right)=a_{2}^{q^{2}+1} \mathrm{~N}\left(a_{3}\right)$, or $b_{2}^{q^{2}+1} \mathrm{~N}\left(b_{3}\right)=\mathrm{N}\left(a_{1}\right) a_{2}^{q^{2}+1}$, respectively. Applying $b_{2}^{q^{2}+1}=a_{2}^{q^{2}+1}$ we get $\mathrm{N}\left(b_{3}\right)=\mathrm{N}\left(a_{3}\right)$, or $\mathrm{N}\left(b_{3}\right)=\mathrm{N}\left(a_{1}\right)$, respectively. Note that the set of elements with norm 1 in $\mathbb{F}_{q^{4}}$ is $\left\{x^{q^{3}-1}: x \in \mathbb{F}_{q^{4}}^{*}\right\}$, thus in the first case there exists $\lambda \in \mathbb{F}_{q^{4}}^{*}$ such that $b_{3}=a_{3} \lambda^{q^{3}-1}$. Then $b_{2} b_{3}^{q^{2}+q}=$ $a_{2} a_{3}^{q^{2}+q}$ yields $b_{2}=a_{2} \lambda^{q^{2}-1}$ and hence $g(x)=a_{0} x+a_{2} \lambda^{q^{2}-1} x^{q^{2}}+a_{3} \lambda^{q^{3}-1} x^{q^{3}}$. 
In the second case the same reasoning yields $g(x)=a_{0} x+a_{2}^{q^{2}} \lambda^{q^{2}-1} x^{q^{2}}+$ $a_{1}^{q^{3}} \lambda^{q^{3}-1} x^{q^{3}}$.

If $b_{2} \neq 0$ and $b_{3}=0$, then the coefficient of $x^{q}$ in $\hat{g}(x)$ is zero and the assertion follows from the above arguments applied to $\hat{g}$ instead of $g$.

Now assume $b_{2}=0$ and $b_{1} b_{3}=0$. Then $L_{g}=L_{f}$ is a linear set of pseudoregulus type and hence the assertion also follows from [17]. For the sake of completeness we present a proof also in this case. Equation $b_{2}^{q^{2}+1}=$ $a_{2}^{q^{2}+1}$ yields $a_{2}=0$ and equation $a_{1} a_{3}^{q}=b_{1} b_{3}^{q}$ yields $a_{1} a_{3}=0$. Then from Lemma 4.1 we have

$$
\mathrm{N}\left(a_{1}\right)+\mathrm{N}\left(a_{3}\right)=\mathrm{N}\left(b_{1}\right)+\mathrm{N}\left(b_{3}\right) .
$$

If $b_{1}=0$, then $b_{3} \neq 0$ and either $a_{1}=0$ and $\mathrm{N}\left(a_{3}\right)=\mathrm{N}\left(b_{3}\right)$, or $a_{3}=0$ and $\mathrm{N}\left(a_{1}\right)=\mathrm{N}\left(b_{3}\right)$. In the first case $g(x)=a_{0} x+a_{3} \lambda^{q^{3}-1} x^{q^{3}}$, in the second case $g(x)=a_{0} x+a_{1}^{q} \lambda^{q^{3}-1} x^{q^{3}}$. If $b_{3}=0$, then $b_{1} \neq 0$ and either $a_{1}=0$ and $\mathrm{N}\left(a_{3}\right)=\mathrm{N}\left(b_{1}\right)$, or $a_{3}=0$ and $\mathrm{N}\left(a_{1}\right)=\mathrm{N}\left(b_{1}\right)$. In the first case $g(x)=a_{0} x+a_{3}^{q} \lambda^{q-1} x^{q}$, in the second case $g(x)=a_{0} x+a_{1} \lambda^{q-1} x^{q}$.

There is only one case left, when $b_{2}=0$ and $b_{1} b_{3} \neq 0$. Then from Lemma 4.1 and from $a_{1} a_{3}^{q}=b_{1} b_{3}^{q}$ it follows that

$$
\mathrm{N}\left(a_{1}\right)+\mathrm{N}\left(a_{3}\right)=\mathrm{N}\left(b_{1}\right)+\mathrm{N}\left(b_{3}\right) .
$$

Together with $(18)$ it follows that either $\mathrm{N}\left(a_{1}\right)=\mathrm{N}\left(b_{1}\right)$ and $\mathrm{N}\left(a_{3}\right)=\mathrm{N}\left(b_{3}\right)$, or $\mathrm{N}\left(a_{1}\right)=\mathrm{N}\left(b_{3}\right)$ and $\mathrm{N}\left(a_{3}\right)=\mathrm{N}\left(b_{1}\right)$. In the first case $g(x)=a_{0} x+$ $a_{1} \lambda^{q-1} x^{q}+a_{3} \lambda^{q^{3}-1} x^{q^{3}}$, in the second case $g(x)=a_{0} x+a_{3}^{q} \lambda^{q-1} x^{q}+a_{1}^{q^{3}} \lambda^{q^{3}-1} x^{q^{3}}$, for some $\lambda \in \mathbb{F}_{q^{4}}^{*}$.

Now we are able to prove the following.

Theorem 4.3. Let $L_{U}$ be an $\mathbb{F}_{q}$-linear set of a line $\mathrm{PG}\left(W, \mathbb{F}_{q^{4}}\right)$ of rank 4 , with maximum field of linearity $\mathbb{F}_{q}$, and let $\beta$ be any non-degenerate alternating form of $W$ over $\mathbb{F}_{q^{4}}$. If $V$ is an $\mathbb{F}_{q}$-vector subspace of $W$ such that $L_{U}=L_{V}$, then either

$$
V=\mu U
$$

or

$$
V=\mu U^{\perp_{\beta}^{\prime}},
$$

for some $\mu \in \mathbb{F}_{q^{4}}^{*}$, where $\perp_{\beta}^{\prime}$ is the orthogonal complement map induced by $\operatorname{Tr}_{q^{4} / q} \circ \beta$ on the lattice of the $\mathbb{F}_{q}$-subspaces of $W$. 
Proof. Assume w.l.o.g. that $L_{U}=L_{V}$ does not contain the point $\langle(0,1)\rangle_{\mathbb{F}_{q^{4}}}$. Then $U=U_{f}$ and $V=V_{g}$ for some $q$-polynomials $f$ and $g$ over $\mathbb{F}_{q^{4}}$. By Proposition 4.2, taking also (6) into account, it follows that there exists $\lambda \in \mathbb{F}_{q^{4}}^{*}$ such that either $\lambda V=U$ or $\lambda V=U^{\perp^{\prime}}$, where $\perp^{\prime}$ is the orthogonal complement map induced by the non-degenerate alternating form $\eta^{\prime}=\operatorname{Tr}_{q^{4} / q}$ ○ $\eta$, with $\eta$ defined in (4). In the first case we have that $V=\mu U$, where $\mu=\frac{1}{\lambda}$. In the second case we have $V=\frac{1}{\lambda} U^{\perp^{\prime}}$. Since $\beta$ and $\eta$ are two non-denegerate alternating forms of the 2-dimensional $\mathbb{F}_{q^{4}}$-space $W$, it follows that there exists $a \in \mathbb{F}_{q^{4}}^{*}$ such that $\beta(\mathbf{x}, \mathbf{y})=a \eta(\mathbf{x}, \mathbf{y})$ for each $\mathbf{x}, \mathbf{y} \in W$. Hence, straightforward computations show that $U^{\perp^{\prime}}=a U^{\perp^{\prime}}$. The assertion follows with $\mu=\frac{a}{\lambda}$.

\subsection{Semilinear maps between $U_{f}$ and $U_{\hat{f}}$}

The next result is just Proposition 3.9 with $n=4$.

Corollary 4.4. Let $f(x)=a_{0} x+a_{1} x^{q}+a_{2} x^{q^{2}}+a_{3} x^{q^{3}}$. There is an $\mathbb{F}_{q^{4}-}$ semilinear map between $U_{f}$ and $U_{\hat{f}}$ if and only if the following system of four equations has a solution $A, B, C, D \in \mathbb{F}_{q^{4}}, A D-B C \neq 0, \sigma=p^{k}$.

$$
\begin{gathered}
C+D a_{0}^{\sigma}-a_{0} A=B a_{0} a_{0}^{\sigma}+\left(B a_{1} a_{1}^{\sigma}\right)^{q^{3}}+\left(B a_{2} a_{2}^{\sigma}\right)^{q^{2}}+\left(B a_{3} a_{3}^{\sigma}\right)^{q}, \\
D a_{1}^{\sigma}-\left(a_{3} A\right)^{q}=B a_{0} a_{1}^{\sigma}+\left(B a_{1} a_{2}^{\sigma}\right)^{q^{3}}+\left(B a_{2} a_{3}^{\sigma}\right)^{q^{2}}+\left(B a_{3} a_{0}^{\sigma}\right)^{q}, \\
D a_{2}^{\sigma}-\left(a_{2} A\right)^{q^{2}}=B a_{0} a_{2}^{\sigma}+\left(B a_{1} a_{3}^{\sigma}\right)^{q^{3}}+\left(B a_{2} a_{0}^{\sigma}\right)^{q^{2}}+\left(B a_{3} a_{1}^{\sigma}\right)^{q} \\
D a_{3}^{\sigma}-\left(a_{1} A\right)^{q^{3}}=B a_{0} a_{3}^{\sigma}+\left(B a_{1} a_{0}^{\sigma}\right)^{q^{3}}+\left(B a_{2} a_{1}^{\sigma}\right)^{q^{2}}+\left(B a_{3} a_{2}^{\sigma}\right)^{q} .
\end{gathered}
$$

Theorem 4.5. Linear sets of rank 4 of $\mathrm{PG}\left(1, q^{4}\right)$, with maximum field of linearity $\mathbb{F}_{q}$, are simple.

Proof. Let $f=\sum_{i=0}^{3} a_{i} x^{q^{i}}$. After a suitable projectivity we may assume $a_{0}=0$. We will use Corollary 4.4 with $\sigma \in\left\{1, q^{2}\right\}$. We may assume that $a_{1}=0$ and $a_{3}=0$ do not hold at the same time since otherwise $f$ is $\mathbb{F}_{q^{2-}}$ linear.

First consider the case when $\mathrm{N}\left(a_{1}\right)=\mathrm{N}\left(a_{3}\right)$. Let $B=C=0, D=A^{q^{2}}$ and take $A$ such that $A^{q-1}=a_{3} / a_{1}^{q}$. This can be done since $\mathrm{N}\left(a_{3} / a_{1}^{q}\right)=1$. Then Corollary 4.4 with $\sigma=q^{2}$ provides the existence of an $\mathbb{F}_{q^{4}}$-semilinear map between $U_{f}$ and $U_{\hat{f}}$.

From now on we assume $\mathrm{N}\left(a_{1}\right) \neq \mathrm{N}\left(a_{3}\right)$.

If $a_{2}=a_{1}=0$, then let $\sigma=1, A=D=0, B=1$ and $C=a_{3}^{2 q}$. If $a_{2}=a_{3}=0$, then let $\sigma=1, A=D=0, B=1$ and $C=a_{1}^{2 q^{3}}$. 
Now consider the case $a_{2}=0$ and $a_{1} a_{3} \neq 0$. Let $A=D=0$. Then the equations of Corollary 4.4 with $\sigma=1$ yield

$$
\begin{gathered}
C=B^{q^{3}} a_{1}^{2 q^{3}}+B^{q} a_{3}^{2 q}, \\
0=B^{q} a_{1}^{q} a_{3}^{q}+B^{q^{3}} a_{1}^{q^{3}} a_{3}^{q^{3}} .
\end{gathered}
$$

(23) is equivalent to $0=\left(B a_{1} a_{3}\right)^{q^{2}}+B a_{1} a_{3}$. Since $X^{q^{2}}+X=0$ has $q^{2}$ solutions in $\mathbb{F}_{q^{4}}$, for any $a_{1}$ and $a_{3}$ we can find $B \in \mathbb{F}_{q^{4}}^{*}$ such that 23) is satisfied. If $B^{q^{3}} a_{1}^{2 q^{3}}+B^{q} a_{3}^{2 q} \neq 0$, then let $C$ be this field element. We show that this is always the case. Suppose, contrary to our claim, that $B^{q^{3}-q}=$ $-a_{3}^{2 q} / a_{1}^{2 q^{3}}$. Because of the choice of $B(23)$ yields $B^{q^{3}-q}=-a_{1}^{q-q^{3}} a_{3}^{q-q^{3}}$. Since $B \neq 0$ this implies

$$
-a_{3}^{2 q} / a_{1}^{2 q^{3}}=-a_{1}^{q-q^{3}} a_{3}^{q-q^{3}}
$$

and hence $a_{1}^{q^{2}+1}=a_{3}^{q^{2}+1}$. A contradiction since $\mathrm{N}\left(a_{1}\right) \neq \mathrm{N}\left(a_{3}\right)$. From now on we assume $a_{2} \neq 0$, we may also assume $a_{2}=1$ after a suitable projectivity.

Corollary 4.4 with $\sigma=1$ yields

$$
\begin{gathered}
C=\left(B a_{1}^{2}\right)^{q^{3}}+B^{q^{2}}+\left(B a_{3}^{2}\right)^{q}, \\
D a_{1}-\left(a_{3} A\right)^{q}=\left(B a_{1}\right)^{q^{3}}+\left(B a_{3}\right)^{q^{2}}, \\
D-A^{q^{2}}=\left(B a_{1} a_{3}\right)^{q^{3}}+\left(B a_{3} a_{1}\right)^{q}, \\
D a_{3}-\left(a_{1} A\right)^{q^{3}}=\left(B a_{1}\right)^{q^{2}}+\left(B a_{3}\right)^{q} .
\end{gathered}
$$

The right hand side of $(25)$ is the $q$-th power of the right hand side of (27) and hence $D^{q} a_{3}^{q}-a_{1} A=D a_{1}-a_{3}^{q} A^{q}$, i.e.

$$
a_{3}^{q}(D+A)^{q}=a_{1}(D+A) .
$$

Since $a_{1}$ or $a_{3}$ is non-zero, we have either $D=-A$, or $(D+A)^{q-1}=a_{1} / a_{3}^{q}$. The latter case can be excluded since in that case $\mathrm{N}\left(a_{1}\right)=\mathrm{N}\left(a_{3}\right)$. Let $D=-A$. Then the left hand side of 25 is $w(A):=-A a_{1}-a_{3}^{q} A^{q}$. The kernel of $w$ is trivial and hence $B$ uniquely determines $A$. The inverse of $w$ is

$$
w^{-1}(x)=\frac{-x a_{1}^{q+q^{2}+q^{3}}+x^{q} a_{1}^{q^{2}+q^{3}} a_{3}^{q}-x^{q^{2}} a_{1}^{q^{3}} a_{3}^{q+q^{2}}+x^{q^{3}} a_{3}^{q+q^{2}+q^{3}}}{\mathrm{~N}\left(a_{1}\right)-\mathrm{N}\left(a_{3}\right)} .
$$


Denote the right hand side of $(25)$ by $r(B)$, the right hand side of 26 by $t(B)$. Then $B$ has to be in the kernel of

$$
K(x):=w^{-1}(r(x))+\left(w^{-1}(r(x))\right)^{q^{2}}+t(x) .
$$

If $B=0$, then $A=B=D=0$ and hence this is not a suitable solution. It is easy to see that $\operatorname{Im} t \subseteq \mathbb{F}_{q^{2}}$ and hence also $\operatorname{Im} K \subseteq \mathbb{F}_{q^{2}}$, so the kernel of $K$ has at least dimension 2 .

Let $B \in \operatorname{ker} K, B \neq 0, A:=w^{-1}(r(B))$ and $C:=\left(B a_{1}^{2}\right)^{q^{3}}+B^{q^{2}}+\left(B a_{3}^{2}\right)^{q}$ (we recall $D=-A$ ). This gives a solution. We have to check that $B$ can be chosen such that $A D-B C \neq 0$, i.e.

$$
Q(B):=\left(w^{-1}(r(B))\right)^{2}+B\left(\left(B a_{1}^{2}\right)^{q^{3}}+B^{q^{2}}+\left(B a_{3}^{2}\right)^{q}\right)
$$

is non-zero. We have $w^{-1}(r(x))\left(\mathrm{N}\left(a_{1}\right)-\mathrm{N}\left(a_{3}\right)\right)=\sum_{i=0}^{3} c_{i} x^{q^{i}}$, where

$$
\begin{gathered}
c_{0}=a_{1}^{1+q^{2}+q^{3}} a_{3}^{q}-a_{1}^{q^{3}} a_{3}^{1+q+q^{2}}, \\
c_{1}=a_{3}^{2 q+q^{2}+q^{3}}-a_{1}^{q+q^{3}} a_{3}^{q+q^{2}}, \\
c_{2}=a_{3}^{q+q^{2}+q^{3}} a_{1}^{q^{2}}-a_{1}^{q+q^{2}+q^{3}} a_{3}^{q^{2}}, \\
c_{3}=a_{1}^{q^{2}+q^{3}} a_{3}^{q+q^{3}}-a_{1}^{q+q^{2}+2 q^{3}} .
\end{gathered}
$$

If $X_{0}, X_{1}, X_{2}, X_{3}$ denote the coordinate functions in $\mathrm{PG}\left(3, q^{4}\right)$ and $Q(B)=0$ for some $B \in \mathbb{F}_{q^{4}}$, then the point $\left\langle\left(B, B^{q}, B^{q^{2}}, B^{q^{3}}\right)\right\rangle_{q^{4}}$ is contained in the the quadric $\mathcal{Q}$ of $\mathrm{PG}\left(3, q^{4}\right)$ defined by the equation

$$
\left(\sum_{i=0}^{3} c_{i} X_{i}\right)^{2}+X_{0}\left(X_{1} a_{3}^{2 q}+X_{2}+X_{3} a_{1}^{2 q^{3}}\right)\left(\mathrm{N}\left(a_{1}\right)-\mathrm{N}\left(a_{3}\right)\right)^{2}=0 .
$$

We can see that the equation of $\mathcal{Q}$ is the linear combination of the equations of two degenerate quadrics, a quadric of rank 1 and a quadric of rank 2 . It follows that $\mathcal{Q}$ is always singular and it has rank 2 or 3 . In particular, the rank of $\mathcal{Q}$ is 2 when the intersection of the planes $\mathcal{A}: X_{0}=0$ and $\mathcal{B}: X_{1} a_{3}^{2 q}+X_{2}+X_{3} a_{1}^{2 q^{3}}=0$ is contained in the plane $\mathcal{C}: \sum_{i=0}^{3} c_{i} X_{i}=0$. Straightforward calculations show that under our hypothesis $\left(a_{1} \neq 0\right.$ or $\left.a_{3} \neq 0, \mathrm{~N}\left(a_{1}\right) \neq \mathrm{N}\left(a_{3}\right)\right)$ this happens if and only if $1=a_{1}^{q} a_{3}$.

We recall that the kernel of $K$ has dimension at least two. Let

$$
H=\left\{\left\langle\left(x, x^{q}, x^{q^{2}}, x^{q^{3}}\right)\right\rangle_{q^{4}}: K(x)=0\right\} .
$$


Our aim is to prove that $H$ has points not belonging to the quadric $\mathcal{Q}$, i.e. $H \nsubseteq \mathcal{Q}$.

Note that $x \in \mathbb{F}_{q^{4}} \mapsto\left(x, x^{q}, x^{q^{2}}, x^{q^{3}}\right) \in \mathbb{F}_{q^{4}}^{4}$ is a vector-space isomorphism between $\mathbb{F}_{q^{4}}$ and the 4-dimensional $\mathbb{F}_{q^{\text {-space }}}\left\{\left(x, x^{q}, x^{q^{2}}, x^{q^{3}}\right): x \in \mathbb{F}_{q^{4}}\right\} \subset$ $\mathbb{F}_{q^{4}}^{4}$. Denote by $\bar{H}$ the $\mathbb{F}_{q^{4}}$-extension of $H$, i.e. the projective subspace of $\mathrm{PG}\left(3, q^{4}\right)$ generated by the points of $H$. Then the projective dimension of $\bar{H}$ is dim ker $K-1$. Let $\xi$ denotes the collineation $\left(X_{0}, X_{1}, X_{2}, X_{3}\right) \mapsto$ $\left(X_{3}^{q}, X_{0}^{q}, X_{1}^{q}, X_{2}^{q}\right)$ of $\mathrm{PG}\left(3, q^{4}\right)$. Then the points of $H$ are fixed points of $\xi$ and hence $\xi$ fixes the subspace $\bar{H}$. Note that the subspace of singular points of $\mathcal{Q}$ is always disjoint from $H$ since it is contained in $\mathcal{A}$, while $H$ is disjoint from it.

First of all note that if $\operatorname{dim} \operatorname{ker} K=4$, i.e. $K$ is the zero polynomial, then $H$ is a subgeometry of $\mathrm{PG}\left(3, q^{4}\right)$ isomorphic to $\mathrm{PG}(3, q)$, which clearly cannot be contained in $\mathcal{Q}$. It follows that $\operatorname{dim} \operatorname{ker} K$ is either 3 or 2 , i.e. $H$ is either a $q$-order subplane or a $q$-order subline.

First assume $1 \neq a_{1}^{q} a_{3}$, i.e. the case when $\mathcal{Q}$ has rank 3 . If $H$ is a $q$-order subplane, then $H$ cannot be contained in $\mathcal{Q}$. To see this, suppose the contrary and take three non-concurrent $q$-order sublines of $H$. The

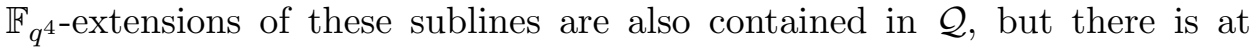
least one of them which does not pass through the singular point of $\mathcal{Q}$, a contradiction. Now assume that $H$ is a $q$-order subline. The singular point of $\mathcal{Q}$ is the intersection of the planes $\mathcal{A}, \mathcal{B}$ and $\mathcal{C}$. Straightforward calculations show that this point is $V=\left\langle\left(v_{0}, v_{1}, v_{2}, v_{3}\right)\right\rangle_{q^{4}}$, where

$$
\begin{gathered}
v_{0}=0, \\
v_{1}=a_{1}^{q^{2}+q^{3}}\left(a_{1}^{q^{3}} a_{3}^{q^{2}}-1\right), \\
v_{2}=a_{1}^{q^{3}} a_{3}^{q}\left(a_{1}^{q^{2}} a_{3}^{q}-a_{1}^{q^{3}} a_{3}^{q^{2}}\right), \\
v_{3}=a_{3}^{q+q^{2}}\left(1-a_{1}^{q^{2}} a_{3}^{q}\right) .
\end{gathered}
$$

Suppose, contrary to our claim, that $H$ is contained in $\mathcal{Q}$. Then $\bar{H}$ passes through the singular point $V$ of $\mathcal{Q}$. Since $\bar{H}$ is fixed by $\xi$, it follows that the points $V, V^{\xi}, V^{\xi^{2}}, V^{\xi^{3}}$ have to be collinear ( $v_{0}=0$ yields that these four points cannot coincide). Let $M$ denote the $4 \times 4$ matrix, whose $i$-th row consists of the coordinates of $V^{\xi^{i-1}}$ for $i=1,2,3,4$. The rank of $M$ is two, thus each of its minors of order three is zero. Let $M_{i, j}$ denote the submatrix of $M$ obtained by deleting the $i$-th row and $j$-th column of $M$. Then

$$
\operatorname{det} M_{1,2}=a_{1}^{q+1}\left(a_{1}^{q} a_{3}-1\right)^{q^{3}+1} \alpha,
$$




$$
\operatorname{det} M_{1,4}=a_{3}^{q^{3}+1}\left(a_{1}^{q} a_{3}-1\right)^{q^{3}+1} \beta,
$$

where

$$
\begin{aligned}
& \alpha=\mathrm{N}\left(a_{1}\right)\left(a_{1}^{q^{2}} a_{3}^{q}-1\right)+\mathrm{N}\left(a_{3}\right)\left(1-a_{1}^{q} a_{3}-a_{1}^{q^{3}} a_{3}^{q^{2}}+a_{1} a_{3}^{q^{3}}\right), \\
& \beta=\mathrm{N}\left(a_{1}\right)\left(a_{1} a_{3}^{q^{3}}+a_{1}^{q^{2}} a_{3}^{q}-a_{1}^{q} a_{3}-1\right)+\mathrm{N}\left(a_{3}\right)\left(1-a_{1}^{q^{3}} a_{3}^{q^{2}}\right) .
\end{aligned}
$$

Since $a_{1}$ and $a_{3}$ cannot be both zeros and $a_{1}^{q} a_{3}-1 \neq 0$, we have $\alpha=\beta=0$.

But $\alpha-\beta=\left(\mathrm{N}\left(a_{1}\right)-\mathrm{N}\left(a_{3}\right)\right)\left(a_{1}^{q} a_{3}-a_{1} a_{3}^{q^{3}}\right)$. It follows that $a_{1}^{q} a_{3} \in \mathbb{F}_{q}$ and hence $\alpha$ can be written as $\left(\mathrm{N}\left(a_{1}\right)-\mathrm{N}\left(a_{3}\right)\right)\left(a_{1}^{q} a_{3}-1\right)$, which is non-zero. This contradiction shows that $V$ cannot be contained in a line fixed by $\xi$ and hence $\bar{H}$ cannot pass through $V$. It follows that $H \nsubseteq \mathcal{Q}$ and hence we can choose $B$ such that $A D-B C \neq 0$.

Now consider the case $1=a_{1}^{q} a_{3}$. Then $\mathcal{Q}$ is the union of two planes meeting each other in $\ell:=\mathcal{A} \cap \mathcal{B}$. It is easy to see that $R:=\left\langle\left(0,1,-a_{3}^{2 q}, 0\right)\right\rangle_{q^{4}}$ and $R^{\xi}$ are two distinct points of $\ell$. Since $\mathrm{N}\left(a_{1}\right) \neq \mathrm{N}\left(a_{3}\right)$ and $\mathrm{N}\left(a_{1}\right) \mathrm{N}\left(a_{3}\right)=$ $1, \operatorname{det}\left\{R, R^{\xi}, R^{\xi^{2}}, R^{\xi^{3}}\right\}=\mathrm{N}\left(a_{3}\right)^{2}-1$ cannot be zero and hence $R \notin H$, otherwise $\operatorname{dim}\left\langle R, R^{\xi}, R^{\xi^{2}}, R^{\xi^{3}}\right\rangle \leq \operatorname{dim} \bar{H} \leq 2$. Suppose, contrary to our claim, that $H$ is contained in one of the two planes of $\mathcal{Q}$. Since $R \notin H$, such a plane can be written as $\langle H, R\rangle$ and since $H$ is fixed by $\xi$ and $\ell \subseteq\langle H, R\rangle$, we have $\langle H, R\rangle^{\xi}=\left\langle H, R^{\xi}\right\rangle=\langle H, R\rangle$. Thus $R, R^{\xi}, R^{\xi^{2}}, R^{\xi^{3}}$ are coplanar, a contradiction.

\section{Different aspects of the classes of a linear set}

\subsection{Class of a linear set and the associated variety}

Let $L_{U}$ be an $\mathbb{F}_{q}$-linear set of rank $k$ of $\mathrm{PG}\left(W, \mathbb{F}_{q^{n}}\right)=\operatorname{PG}\left(r-1, q^{n}\right)$. Consider the projective space $\Omega=\operatorname{PG}\left(W, \mathbb{F}_{q}\right)=\operatorname{PG}(r n-1, q)$. For each point $P=$ $\langle\mathbf{u}\rangle_{\mathbb{F}_{q^{n}}}$ of $\operatorname{PG}\left(W, \mathbb{F}_{q^{n}}\right)$ there corresponds a projective $(n-1)$-subspace $X_{P}:=$ $\operatorname{PG}\left(\langle\mathbf{u}\rangle_{q^{n}}, \mathbb{F}_{q}\right)$ of $\Omega$. The variety of $\Omega$ associated to $L_{U}$ is

$$
\mathcal{V}_{r, n, k}\left(L_{U}\right)=\bigcup_{P \in L_{U}} X_{P}
$$

This variety was already used in [2] and [17], see Example 5.1. The question of determining whether a linear set is simple or not is related to the existence of so-called irregular subspaces (see [17]). The case of irregular sublines was already studied in [11.

A $(k-1)$-space $\mathcal{H}=\operatorname{PG}\left(V, \mathbb{F}_{q}\right)$ of $\Omega$ is said to be a transversal space of $\mathcal{V}\left(L_{U}\right)$ if $\mathcal{H} \cap X_{P} \neq \emptyset$ for each point $P \in L_{U}$, i.e. $L_{U}=L_{V}$. 
The $\mathcal{Z}(\Gamma \mathrm{L})$-class of an $\mathbb{F}_{q^{-}}$-linear set $L_{U}$ of rank $n$ of $\mathrm{PG}\left(W, \mathbb{F}_{q^{n}}\right)=$ $\operatorname{PG}\left(1, q^{n}\right)$, with maximum field of linearity $\mathbb{F}_{q}$, is the number of transversal

spaces of $\mathcal{V}_{2, n, n}\left(L_{U}\right)$ up to the action of the subgroup $G$ of $\operatorname{PGL}(2 n-1, q)$ induced by the maps $\mathbf{x} \in W \mapsto \lambda \mathbf{x} \in W$, with $\lambda \in \mathbb{F}_{q^{n}}^{*}$. Note that $G$ fixes $X_{P}$ for each point $P \in \mathrm{PG}\left(1, q^{n}\right)$ and hence fixes the variety.

The maximum size of an $\mathbb{F}_{q^{-}}$-linear set $L_{U}$ of rank $n$ of $\operatorname{PG}\left(1, q^{n}\right)$ is $\left(q^{n}-1\right) /(q-1)$. If this bound is attained (hence each point of $L_{U}$ has weight one), then $L_{U}$ is a maximum scattered linear set of $\operatorname{PG}\left(1, q^{n}\right)$. For maximum scattered linear sets, the number of transversal spaces through $Q \in \mathcal{V}\left(L_{U}\right)$ does not depend on the choice of $Q$ and this number is the $\mathcal{Z}(\Gamma \mathrm{L})$-class of $L_{U}$.

Example 5.1. Let $U=\left\{\left(x, x^{q}\right): x \in \mathbb{F}_{q^{n}}\right\}$ and consider the linear set $L_{U}$. In [17] the variety $\mathcal{V}_{2, n, n}\left(L_{U}\right)$ was studied, and the transversal spaces were determined. It follows that the $\mathcal{Z}(\Gamma \mathrm{L})$-class of $L_{U}$ is $\varphi(n)$, where $\varphi$ is the Euler's phi function.

\subsection{Classes of linear sets as projections of subgeometries}

Let $\Sigma=\mathrm{PG}(k-1, q)$ be a canonical subgeometry of $\Sigma^{*}=\mathrm{PG}\left(k-1, q^{n}\right)$. Let $\Gamma \subset \Sigma^{*} \backslash \Sigma$ be a $(k-r-1)$-space and let $\Lambda \subset \Sigma^{*} \backslash \Gamma$ be an $(r-1)$-space of $\Sigma^{*}$. The projection of $\Sigma$ from center $\Gamma$ to axis $\Lambda$ is the point set

$$
L=p_{\Gamma, \Lambda}(\Sigma):=\{\langle\Gamma, P\rangle \cap \Lambda: P \in \Sigma\} .
$$

In 24] Lunardon and Polverino characterized linear sets as projections of canonical subgeometries. They proved the following.

Theorem 5.2 ([24, Theorems 1 and 2]). Let $\Sigma^{*}, \Sigma, \Lambda, \Gamma$ and $L=p_{\Gamma, \Lambda}(\Sigma)$ be defined as above. Then $L$ is an $\mathbb{F}_{q}$-linear set of rank $k$ and $\langle L\rangle=\Lambda$. Conversely, if $L$ is an $\mathbb{F}_{q}$-linear set of rank $k$ of $\Lambda=\mathrm{PG}\left(r-1, q^{n}\right) \subset \Sigma^{*}$ and $\langle L\rangle=\Lambda$, then there is a $(k-r-1)$-space $\Gamma$ disjoint from $\Lambda$ and a canonical subgeometry $\Sigma=\operatorname{PG}(r-1, q)$ disjoint from $\Gamma$ such that $L=p_{\Gamma, \Lambda}(\Sigma)$.

Let $L_{U}$ be an $\mathbb{F}_{q^{-}}$-linear set of rank $k$ of $\mathbb{P}=\operatorname{PG}\left(W, \mathbb{F}_{q^{n}}\right)=\operatorname{PG}\left(r-1, q^{n}\right)$ such that for each $k$-dimensional $\mathbb{F}_{q}$-subspace $V$ of $W$ if $\operatorname{PG}\left(V, \mathbb{F}_{q}\right)$ is a transversal space of $\mathcal{V}_{r, n, k}\left(L_{U}\right)$, then there exists $\gamma \in \operatorname{P\Gamma L}\left(W, \mathbb{F}_{q}\right)$, such that $\gamma$ fixes the Desarguesian spread $\left\{X_{P}: P \in \mathbb{P}\right\}$ and $\operatorname{PG}\left(U, \mathbb{F}_{q}\right)^{\gamma}=\operatorname{PG}\left(V, \mathbb{F}_{q}\right)$. This is condition (A) from [7, and it is equivalent to say that $L_{U}$ is a simple linear set. Then the main results of [7] can be formalized as follows.

Theorem 5.3 ([7]). Let $L_{1}=p_{\Gamma_{1}, \Lambda_{1}}\left(\Sigma_{1}\right)$ and $L_{2}=p_{\Gamma_{2}, \Lambda_{2}}\left(\Sigma_{2}\right)$ be two linear sets of rank $k$. If $L_{1}$ and $L_{2}$ are equivalent and one of them is simple, then there is a collineation mapping $\Gamma_{1}$ to $\Gamma_{2}$ and $\Sigma_{1}$ to $\Sigma_{2}$. 
Theorem 5.4 ([7]). If $L$ is a non-simple linear set of rank $k$ in $\Lambda=\langle L\rangle$, then there are a subspace $\Gamma=\Gamma_{1}=\Gamma_{2}$ disjoint from $\Lambda$, and two q-order canonical subgeometries $\Sigma_{1}, \Sigma_{2}$ such that $L=p_{\Gamma, \Lambda}\left(\Sigma_{1}\right)=p_{\Gamma, \Lambda}\left(\Sigma_{2}\right)$, and there is no collineation fixing $\Gamma$ and mapping $\Sigma_{1}$ to $\Sigma_{2}$.

Now we interpret the classes of linear sets, hence we are going to consider $\mathbb{F}_{q^{-}}$-linear sets of rank $n$ of $\Lambda=\mathrm{PG}\left(1, q^{n}\right)=\mathrm{PG}\left(W, \mathbb{F}_{q^{n}}\right)$, with maximum field of linearity $\mathbb{F}_{q}$. Arguing as in the proof of [7, Theorem 7], if $L_{U}$ is non-simple, then for any pair $U, V$ of $n$-dimensional $\mathbb{F}_{q}$-subspaces of $W$ with $L_{U}=L_{V}$ such that $U^{f} \neq V$ for each $f \in \Gamma L\left(2, q^{n}\right)$ we can find a $q$-order subgeometry $\Sigma$ of $\Sigma^{*}=\operatorname{PG}\left(n-1, q^{n}\right)$ and two $(n-3)$-spaces $\Gamma_{1}$ and $\Gamma_{2}$ of $\Sigma^{*}$, disjoint from $\Sigma$ and from $\Lambda$, lying on different orbits of $\operatorname{Stab}(\Sigma)$. On the other hand, arguing as in [7, Theorem 6], if there exist two $(n-3)$-subspaces $\Gamma_{1}$ and $\Gamma_{2}$ of $\Sigma^{*}$, disjoint from $\Sigma$ and from $\Lambda$, belonging to different orbits of $\operatorname{Stab}(\Sigma)$ and such that $L=p_{\Lambda, \Gamma_{1}}(\Sigma)=p_{\Lambda, \Gamma_{2}}(\Sigma)$, then it is possible to construct two

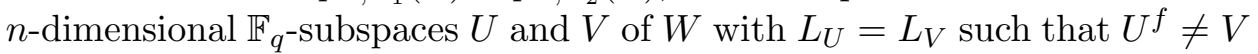
for each $f \in \Gamma \mathrm{L}\left(2, q^{n}\right)$. Hence we can state the following.

The $\Gamma$-class of $L_{U}$ is the number of orbits of $\operatorname{Stab}(\Sigma)$ on $(n-3)$-spaces of $\Sigma^{*}$ containing a $\Gamma$ disjoint from $\Sigma$ and from $\Lambda$ such that $p_{\Lambda, \Gamma}(\Sigma)$ is equivalent to $L_{U}$.

\subsection{Class of linear sets and linear blocking sets of Rédei type}

A blocking set $\mathcal{B}$ of $\operatorname{PG}\left(V, \mathbb{F}_{q^{n}}\right)=\operatorname{PG}\left(2, q^{n}\right)$ is a point set meeting every line of the plane. Blocking sets of size $q^{n}+N \leq 2 q^{n}$ with an $N$-secant are called blocking sets of Rédei type, the $N$-secants of the blocking set are called Rédei lines. Let $L_{U}$ be an $\mathbb{F}_{q}$-linear set of rank $n$ of a line $\ell=\operatorname{PG}\left(W, \mathbb{F}_{q^{n}}\right)$, $W \leq V$, and let $\mathbf{w} \in V \backslash W$. Then $\langle U, \mathbf{w}\rangle_{\mathbb{F}_{q}}$ defines an $\mathbb{F}_{q}$-linear blocking set of $\operatorname{PG}\left(2, q^{n}\right)$ with Rédei line $\ell$. The following theorem tells us the number of inequivalent blocking sets obtained in this way.

Theorem 5.5. The $\Gamma \mathrm{L}$-class of an $\mathbb{F}_{q}$-linear set $L_{U}$ of rank $n$ of $\mathrm{PG}\left(W, \mathbb{F}_{q^{n}}\right)=$ $\mathrm{PG}\left(1, q^{n}\right)$, with maximum field of linearity $\mathbb{F}_{q}$, is the number of inequivalent

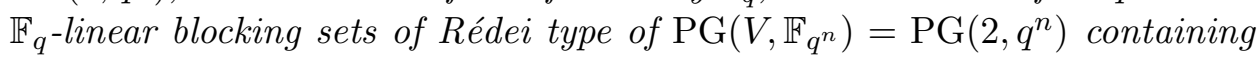
$L_{U}$.

Proof. $\mathbb{F}_{q^{-}}$-linear blocking sets of $\mathrm{PG}\left(2, q^{n}\right)$ with more than one Rédei line are equivalent to those defined by $\operatorname{Tr}_{q^{n}} / q^{m}(x)$ for some divisor $m$ of $n$, see [22, Theorem 5]. Suppose first that $L_{U}$ is equivalent to $L_{T}$, where $T=$ $\left\{\left(x, \operatorname{Tr}_{q^{n} / q}(x)\right): x \in \mathbb{F}_{q^{n}}\right\}$. According to Theorem 3.7 $L_{T}$, and hence also $L_{U}$, have $\mathcal{Z}(\Gamma L)$-class and $\Gamma L$-class one and hence there exists a unique 
point $P \in L_{U}$ such that $w_{L_{U}}(P)=n-1$. Then for each $\mathbf{v} \in V \backslash W$ the $\mathbb{F}_{q^{-}}$ linear blocking set defined by $\langle U, \mathbf{v}\rangle_{\mathbb{F}_{q}}$ has more than one Rédei line, each of them incident with $P$, and hence it is equivalent to the Rédei type blocking set obtained from $\operatorname{Tr}_{q^{n} / q}(x)$.

Now let $\mathcal{B}_{1}=L_{V_{1}}$ and $\mathcal{B}_{2}=L_{V_{2}}$ be two $\mathbb{F}_{q^{-}}$-linear blocking sets of Rédei type with $\operatorname{PG}\left(W, \mathbb{F}_{q^{n}}\right)$ the unique Rédei line. Denote by $U_{1}$ and $U_{2}$ the $\mathbb{F}_{q^{-}}$ subspaces $W \cap V_{1}$ and $W \cap V_{2}$, respectively, and suppose $L_{U_{1}}=L_{U_{2}}$ with $\mathbb{F}_{q}$ the maximum field of linearity. Then $\mathcal{B}_{1}$ and $\mathcal{B}_{2}$ have $(q+1)$-secants and we have $V_{1}=U_{1} \oplus\left\langle\mathbf{u}_{\mathbf{1}}\right\rangle_{\mathbb{F}_{q}}$ and $V_{2}=U_{2} \oplus\left\langle\mathbf{u}_{\mathbf{2}}\right\rangle_{\mathbb{F}_{q}}$ for some $\mathbf{u}_{\mathbf{1}}, \mathbf{u}_{\mathbf{2}} \in V \backslash W$.

If $\mathcal{B}_{1}^{\varphi_{f}}=\mathcal{B}_{2}$, then [6. Proposition 2.3] implies $V_{1}^{f}=\lambda V_{2}$ for some $\lambda \in \mathbb{F}_{q^{n}}^{*}$. Such $f \in \Gamma \mathrm{L}\left(3, q^{n}\right)$ has to fix $W$ and it is easy to see that $U_{1}^{f}=\lambda U_{2}$, i.e. $U_{1}$ and $U_{2}$ are $\Gamma \mathrm{L}\left(2, q^{n}\right)$-equivalent.

Conversely, if there exists $f \in \Gamma \mathrm{L}\left(W, \mathbb{F}_{q^{n}}\right)$ such that $U_{1}^{f}=U_{2}$, then $\mathcal{B}_{1}^{\varphi_{g}}=\mathcal{B}_{2}$, where $g \in \Gamma L\left(V, \mathbb{F}_{q^{n}}\right)$ is the extension of $f$ mapping $\mathbf{u}_{\mathbf{1}}$ to $\mathbf{u}_{\mathbf{2}}$.

\subsection{Class of linear sets and MRD-codes}

In [28, Section 4] Sheekey showed that maximum scattered $\mathbb{F}_{q}$-linear sets

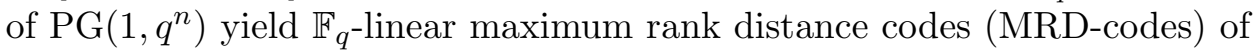
dimension $2 n$ and minimum distance $n-1$, that is, a set $\mathcal{M}$ of $q^{2 n} n \times n$ matrices over $\mathbb{F}_{q}$ forming an $\mathbb{F}_{q}$-subspace of $\mathbb{F}_{q}^{n \times n}$ of dimension $2 n$ such that the non-zero matrices of $\mathcal{M}$ have rank at least $n-1$. It can be easily seen that these MRD-codes have the so-called middle nucleus isomorphic to $\mathbb{F}_{q^{n}}$. For definitions and properties on MRD-codes we refer the reader to [10] by Delsarte and [13] by Gabidulin. The kernel and the nuclei of MRD-codes are studied in [26].

For $n \times n$ matrices there are two different definitions of equivalence for MRD-codes in the literature. The arguments of [28, Section 4] yield the following interpretation of the $\Gamma$ L-class:

- $\mathcal{M}$ and $\mathcal{M}^{\prime}$ are equivalent if there are invertible matrices $A, B \in \mathbb{F}_{q}^{n \times n}$ and a field automorphism $\sigma$ of $\mathbb{F}_{q}$ such that $A \mathcal{M}^{\sigma} B=\mathcal{M}^{\prime}$, see [28]. In this case the $\Gamma$ L-class of $L_{U}$ is the number of inequivalent MRD-codes obtained from the linear set $L_{U}$.

- $\mathcal{M}$ and $\mathcal{M}^{\prime}$ are equivalent if there are invertible matrices $A, B \in$ $\mathbb{F}_{q}^{n \times n}$ and a field automorphism $\sigma$ of $\mathbb{F}_{q}$ such that $A \mathcal{M}^{\sigma} B=\mathcal{M}^{\prime}$, or $A \mathcal{M}^{T \sigma} B=\mathcal{M}^{\prime}$, see [9]. In this case the number of inequivalent MRDcodes obtained from the linear set $L_{U}$ is between $\lceil s / 2\rceil$ and $s$, where $s$ is the $\Gamma$ L-class of $L_{U}$. 
We summarize here the known non-equivalent families of MRD-codes arising from maximum scattered linear sets.

1. $L_{U_{1}}:=\left\{\left\langle\left(x, x^{q}\right)\right\rangle_{\mathbb{F}_{q^{n}}}: x \in \mathbb{F}_{q^{n}}^{*}\right\}([5])$ gives Gabidulin codes,

2. $L_{U_{2}}:=\left\{\left\langle\left(x, x^{q^{s}}\right)\right\rangle_{\mathbb{F}_{q^{n}}}: x \in \mathbb{F}_{q^{n}}^{*}\right\}, \operatorname{gcd}(s, n)=1$ ([5]) gives generalized Gabidulin codes,

3. $L_{U_{3}}:=\left\{\left\langle\left(x, \delta x^{q}+x^{q^{n-1}}\right)\right\rangle_{\mathbb{F}_{q^{n}}}: x \in \mathbb{F}_{q^{n}}^{*}\right\}([23])$ gives MRD-codes found by Sheekey in [28],

4. $L_{U_{4}}:=\left\{\left\langle\left(x, \delta x^{q^{s}}+x^{q^{n-s}}\right)\right\rangle_{\mathbb{F}_{q^{n}}}: x \in \mathbb{F}_{q^{n}}^{*}\right\}, \mathrm{N}(\delta) \neq 1, \operatorname{gcd}(s, n)=1$ gives MRD-codes found by Sheekey in [28] and studied by Lunardon, Trombetti and Zhou in [25].

Remark 5.6. The linear sets $L_{U_{1}}$ and $L_{U_{2}}$ coincide, but when $s \notin\{1, n-1\}$, there is no $f \in \Gamma \mathrm{L}\left(2, q^{n}\right)$ such that $U_{1}^{f}=U_{2}$. These linear sets are of pseudoregulus type, [21] (see also Example 5.1), and in [7] it was proved that the $\Gamma \mathrm{L}$-class of these linear sets is $\varphi(n) / 2$, hence they are examples of non-simple linear sets for $n=5$ and $n>6$.

It can be proved that the family $L_{U_{4}}$ contains linear sets non-equivalent to those from the other families. We will report on this elsewhere.

\section{References}

[1] S. BALL: The number of directions determined by a function over a finite field, J. Combin. Theory Ser. A 104 (2003), 341-350.

[2] S. Ball, A. Blokhuis, M. Lavrauw: Linear $(q+1)$-fold blocking sets in $P G\left(2, q^{4}\right)$, Finite Fields Appl., 6 (2000), 294-301.

[3] S. Ball, A. Blokhuis, A.E. Brouwer, L. Storme and T. SzŐNYI: On the number of slopes of the graph of a function definied over a finite field, J. Combin. Theory Ser. A 86 (1999), 187-196.

[4] D. Bartoli, M. Giulietti, G. Marino and O. Polverino: Maximum scattered linear sets and complete caps in Galois spaces, to appear in Combinatorica. DOI: 10.1007/s00493-016-3531-6.

[5] A. Blokhuis and M. Lavrauw: Scattered spaces with respect to a spread in PG(n,q), Geom. Dedicata 81 (2000), 231-243. 
[6] G. Bonoli and O. Polverino: $\mathbb{F}_{q}$-linear blocking sets in PG $\left(2, q^{4}\right)$, Innov. Incidence Geom. 2 (2005), 35-56.

[7] B. Csajbók and C. Zanella: On the equivalence of linear sets, Des. Codes Cryptogr. 81 (2016), 269-281.

[8] M. De Boeck and G. Van de Voorde: A linear set view on KM-arcs, J. Algebr. Comb. 44, n.1 (2016), 131-164.

[9] J. De la Cruz, M. Kiermaier, A. Wasserman and W. Williems: Algebraic structures of MRD Codes, Adv. Math. Commun. 10 (2016), 499-510.

[10] P. Delsarte: Bilinear forms over a finite field, with applications to coding theory, J. Combin. Theory Ser. A 25 (1978), 226-241.

[11] G. Donati and N. Durante: Scattered linear sets generated by collineations between pencils of lines, J. Algebr. Comb. 40, n. 4 (2014), 1121-1131.

[12] Sz. Fancsali and P. Sziklai: Description of the clubs, Annales Univ. Sci. Sect. Mat. 51 (2008), 141-146.

[13] E. Gabidulin: Theory of codes with maximum rank distance, Problems of information transmission, 21(3) (1985), 3-16.

[14] N. GILL: Polar spaces and embeddings of classical groups, N. Z. J. Math., 36 (2007), 175-184.

[15] J.W.P. Hirschfeld and J.A. Thas: General Galois Geometries. Ofxord University Press, 1991.

[16] M. Lavrauw: Scattered spaces in Galois Geometry, Contemporary Developments in Finite Fields and Applications, 2016, 195-216.

[17] M. Lavrauw, J. Sheekey and C. Zanella: On embeddings of minimum dimension of $\mathrm{PG}(n, q) \times \mathrm{PG}(n, q)$, Des. Codes Cryptogr. 74. n.2 (2015), 427-440.

[18] M. Lavrauw and G. Van de Voorde: On linear sets on a projective line, Des. Codes Cryptogr. 56 (2010), 89-104.

[19] M. Lavrauw and G. Van de Voorde: Field reduction and linear sets in finite geometry, in: Gohar Kyureghyan, Gary L. Mullen, 
Alexander Pott (Eds.), Topics in Finite Fields, Contemp. Math. AMS (2015).

[20] G. Lunardon: Normal spreads, Geom. Dedicata 75 (1999), 245261.

[21] G. Lunardon, G. Marino, O. Polverino and R. Trombetti: Maximum scattered linear sets of pseudoregulus type and the Segre Variety $\mathcal{S}_{n, n}$, J. Algebr. Comb. 39 (2014), 807-831.

[22] G. Lunardon and O. Polverino: Blocking Sets of Size $q^{t}+$ $q^{t-1}+1$, J. Combin. Theory Ser. A 90 (2000), 148-158.

[23] G. Lunardon and O. Polverino: Blocking Sets and Derivable Partial Spreads, J. Algebraic Combin. 14 (2001), 49-56.

[24] G. Lunardon and O. Polverino: Translation ovoids of orthogonal polar spaces, Forum Math. 16 (2004), 663-669.

[25] G. Lunardon, R. Trombetti and Y. Zhou: Generalized Twisted Gabidulin Codes, http://arxiv.org/abs/1507.07855.

[26] G. Lunardon, R. Trombetti and Y. Zhou: On kernels and nuclei of rank metric codes, J. Algebraic Combin. 46 (2017), 313340 .

[27] O. Polverino: Linear sets in finite projective spaces, Discrete Math. 310 (2010), 3096-3107.

[28] J. Sheekey: A new family of linear maximum rank distance codes, Adv. Math. Commun. 10(3) (2016), 475-488.

[29] B. Wu AND Z. LiU:, Linearized polynomials over finite fields revisited, Finite Fields Appl. 22 (2013), 79-100.

Bence Csajbók

MTA-ELTE Geometric and Algebraic Combinatorics Research Group

ELTE Eötvös Loránd University, Budapest, Hungary

Department of Geometry

1117 Budapest, Pázmány P. stny. 1/C, Hungary

csajbok.bence@gmail.com

and 
Dipartimento di Matematica e Fisica,

Università degli Studi della Campania "Luigi Vanvitelli",

Viale Lincoln 5, I- 81100 Caserta, Italy

Giuseppe Marino, Olga Polverino

Dipartimento di Matematica e Fisica,

Università degli Studi della Campania "Luigi Vanvitelli",

Viale Lincoln 5, I- 81100 Caserta, Italy

giuseppe.marino@unicampania.it,olga.polverino@unicampania.it 